\title{
Systematic Review of the Neural Effect of Electroconvulsive Therapy in Patients with Schizophrenia: Hippocampus and Insula as the Key Regions of Modulation
}

\author{
Sun-Young Moon ${ }^{1,2}$, Minah Kim ${ }^{1,2}$, Silvia Kyungjin Lho', \\ Sanghoon $\mathrm{Oh}^{1,2}$, Se Hyun Kim ${ }^{1,2}$, and Jun Soo Kwon ${ }^{1,2,3,4}$ \\ ${ }^{1}$ Department of Psychiatry, Seoul National University College of Medicine, Seoul, Republic of Korea \\ ${ }^{2}$ Department of Neuropsychiatry, Seoul National University Hospital, Seoul, Republic of Korea \\ ${ }^{3}$ Department of Brain and Cognitive Sciences, Seoul National University College of Natural Sciences, Seoul, Republic of Korea \\ ${ }^{4}$ Institute of Human Behavioral Medicine, SNU-MRC, Seoul, Republic of Korea
}

Objective Electroconvulsive therapy (ECT) has been the most potent treatment option for treatment-resistant schizophrenia (TRS). However, the underlying neural mechanisms of ECT in schizophrenia remain largely unclear. This paper examines studies that investigated structural and functional changes after ECT in patients with schizophrenia.

Methods We carried out a systematic review with following terms: 'ECT', 'schizophrenia', and the terms of various neuroimaging modalities.

Results Among the 325 records available from the initial search in May 2020, 17 studies were included. Cerebral blood flow in the frontal, temporal, and striatal structures was shown to be modulated $(n=3)$, although the results were divergent. Magnetic resonance spectroscopy (MRS) studies suggested that the ratio of $\mathrm{N}$-acetyl-aspartate/creatinine was increased in the left prefrontal cortex $(\mathrm{PFC} ; \mathrm{n}=2)$ and left thalamus $(n=1)$. The hippocampus and insula $(n=6$, respectively) were the most common regions of structural/functional modulation, which also showed symptom associations. Functional connectivity of the default mode network (DMN; n=5), PFC ( $=4$ ), and thalamostriatal system $(n=2)$ were also commonly modulated.

Conclusion Despite proven effectiveness, there has been a dearth of studies investigating the neurobiological mechanisms underlying ECT. There is preliminary evidence of structural and functional modulation of the hippocampus and insula, functional changes in the DMN, PFC, and thalamostriatal system after ECT in patients with schizophrenia. We discuss the rationale and implications of these findings and the potential mechanism of action of ECT. More studies evaluating the mechanisms of ECT are needed, which could provide a unique window into what leads to treatment response in the otherwise refractory TRS population. Psychiatry Investig 2021;18(6):486-499

Key Words Electroconvulsive therapy, Schizophrenia, Treatment-resistant schizophrenia, Hippocampus, Insula.

\section{INTRODUCTION}

Schizophrenia affects approximately $1 \%$ of global population, with more than 21 million patients worldwide. ${ }^{1}$ Treatment-resistant schizophrenia (TRS) is defined as the absence of a response after two different antipsychotics at an adequate

\footnotetext{
Received: December 21, 2020 Revised: February 21, 2021 Accepted: March 3, 2021

$\triangle$ Correspondence: Minah Kim, MD, PhD

Department of Neuropsychiatry, Seoul National University Hospital, 101 Daehak-ro, Jongno-gu, Seoul 03080, Republic of Korea

Tel: +82-2-2072-7211, Fax: +82-2-747-9063, E-mail: verte82@snu.ac.kr

(a) This is an Open Access article distributed under the terms of the Creative Commons Attribution Non-Commercial License (https://creativecommons.org/licenses/bync/4.0) which permits unrestricted non-commercial use, distribution, and reproduction in any medium, provided the original work is properly cited.
}

dosage and duration. Twenty to $50 \%$ of patients with schizophrenia are considered treatment-resistant, ${ }^{2-4}$ and, not surprisingly, treatment-resistance results in an additional socioeconomic burden that is approximately 3 to 11 times greater than the burden experienced by patients with schizophrenia who remain in remission. ${ }^{5}$ The treatment of choice for these patients is to introduce the medication clozapine, ${ }^{2,4,6}$ but $40 \%$ to $70 \%$ of treatment-resistant patients will not respond to clozapine, ${ }^{7}$ leaving $14 \%$ to $20 \%$ of all patients with schizophrenia to be treatment refractory without better treatment options. ${ }^{5}$

Electroconvulsive therapy (ECT) was first introduced to treat schizophrenia before antipsychotic medication was available. ${ }^{8}$ After the late 1950s, with the advent of antipsychotic agents, the use of ECT in schizophrenia declined worldwide, ${ }^{9}$ 
with its primary indication being catatonia, psychotic depression, suicidality, and drug intolerability. ${ }^{10}$ Although its main pattern of use somewhat differs among countries (according to a systematic review in 2011, in United States, Europe and Australia, $8-29 \%$ of patients receiving ECT were patients with schizophrenia, while in Asia and Africa it was 60-83\%), ${ }^{9,11,12}$ ECT is currently most commonly used for patients with treatment-resistant depression, ${ }^{11}$ where its efficacy is robust and superior compared to the standard combination of antidepressants. ${ }^{13,14}$ TRS is another clinical group in which ECT is commonly used to augment standard antipsychotic treatment. ${ }^{10,15} \mathrm{~A}$ large volume of clinical research literature and systematic reviews along with an increasing number of treatment guidelines support the addition of ECT to antipsychotic agent regimens, ${ }^{15-23}$ including a recent review from the Cochrane systematic reviews. ${ }^{24}$ Considering the prevalence and rather striking socioeconomic consequences of TRS, clinicians have argued that ECT should not be used as the "last resort' and that it should be increasingly applied to patients with schizophrenia. ${ }^{9.25}$

Despite this context of robust effectiveness, the underlying mechanism of action of ECT has largely remained elusive. Historically, the generalized seizure hypothesis, changes in neurotransmitters and neuroendocrine function hypothesis, and the neurotrophic hypothesis has been postulated as working models of ECT. ${ }^{26}$ Recent studies examining depressive patients have consistently found increased bilateral hippocampal volumes by $\mathrm{ECT}^{27-31}$ providing support for the neurotrophic hypothesis, but correlations between volumetric changes and clinical improvements were found to be repeatedly inconclusive, leaving out the main 'key' factor regarding the long unanswered question of ECT's clinical effectiveness. Furthermore, the factors leading to clinical improvement after ECT in schizophrenia patients are cryptic, with only a small number of studies and divergent findings. This is indicative of a largely underrepresented current state of research for a disease with such great burden and the tantalizing problems of obstinacy. Identification and delineation of the common and disease-specific effects of ECT can serve to provide insights into the currently 'untreatable' TRS population, which constitutes a major problem in the modern practice of psychiatry. Thus, we aimed to systematically review the current status of research investigating the effects of ECT in schizophrenia patients using, utilizing etc. various neuroimaging modalities.

\section{METHODS}

PubMed, EMBASE, and the Cochrane Reviews were searched for publications with the following keywords: 'ECT', 'schizophrenia, and the terms of various neuroimaging modalities.
Studies available based on the search by May 1,2020, were included. We intended to include articles that examined patients with schizophrenia undergoing ECT sessions and implemented at least one neuroimaging modality according to the Preferred Reporting Items for Systematic Reviews and Meta-Analyses (PRISMA) Statement. Articles published before the 1990s were excluded owing to two reasons: 1) to minimize the heterogeneity among study methods, and because 2) earlier studies tended to focus on deleterious side effects of ECT. ${ }^{32-34}$ While ECT can bring about selective cognitive side effects such as acute delirium or impairments in autobiographic memory, it is nowadays widely agreed that ECT does not cause other severe long-lasting side effects such as brain damage or dementia. ${ }^{35}$ Detailed descriptions of the methods are provided in the supplementary methods in the supplementary material.

\section{RESULTS}

\section{Study selection and characteristics}

During the initial search, 325 articles were found, and after removing 3 duplicates, 322 articles were screened. A total of 305 records that unmet the inclusion criteria were excluded after abstract screening (PRISMA flowchart, Figure 1).

A total of 17 studies were selected and considered eligible for inclusion in the current review. Three of the studies explored the role of ECT in changes in cerebral blood flow (CBF), 3 studies used magnetic resonance spectroscopy (MRS) to measure changes in brain metabolites, and 11 studies used magnetic resonance imaging (MRI) as the study modality (6 structural and 8 functional studies, with 3 using both modalities). Detailed information about each study eligible for inclusion in the review is provided in Supplementary Results and Supplementary Table 1 in the Supplementary Materials (in the online-only Data Supplement).

\section{Effect of ECT on cerebral blood flow}

Three studies measured changes in CBF using differing study modalities, namely, of positron emission tomography (PET), single-photon emission computed tomography (SPECT), and near-infrared spectroscopy (NIRS) (Table 1).

A PET study reported decreased CBF in the bilateral frontal lobes, right temporal lobe, and right putamen after ECT $(n=5)$ compared to controls $(n=6) \cdot{ }^{36}$ In another study, CBF in the right parietal and bilateral temporal lobes measured using SPECT was increased in patients with schizophrenia $(n=5)$ compared with MDD patients $(\mathrm{n}=5) \cdot{ }^{37}$ A NIRS study revealed an increased blood flow ratio of the left prefrontal cortex (PFC) to right PFC in patients with schizophrenia $(\mathrm{n}=11)$ after ECT, an asymmetry that was not observed in the MDD $(n=10)$ group. ${ }^{38}$ 


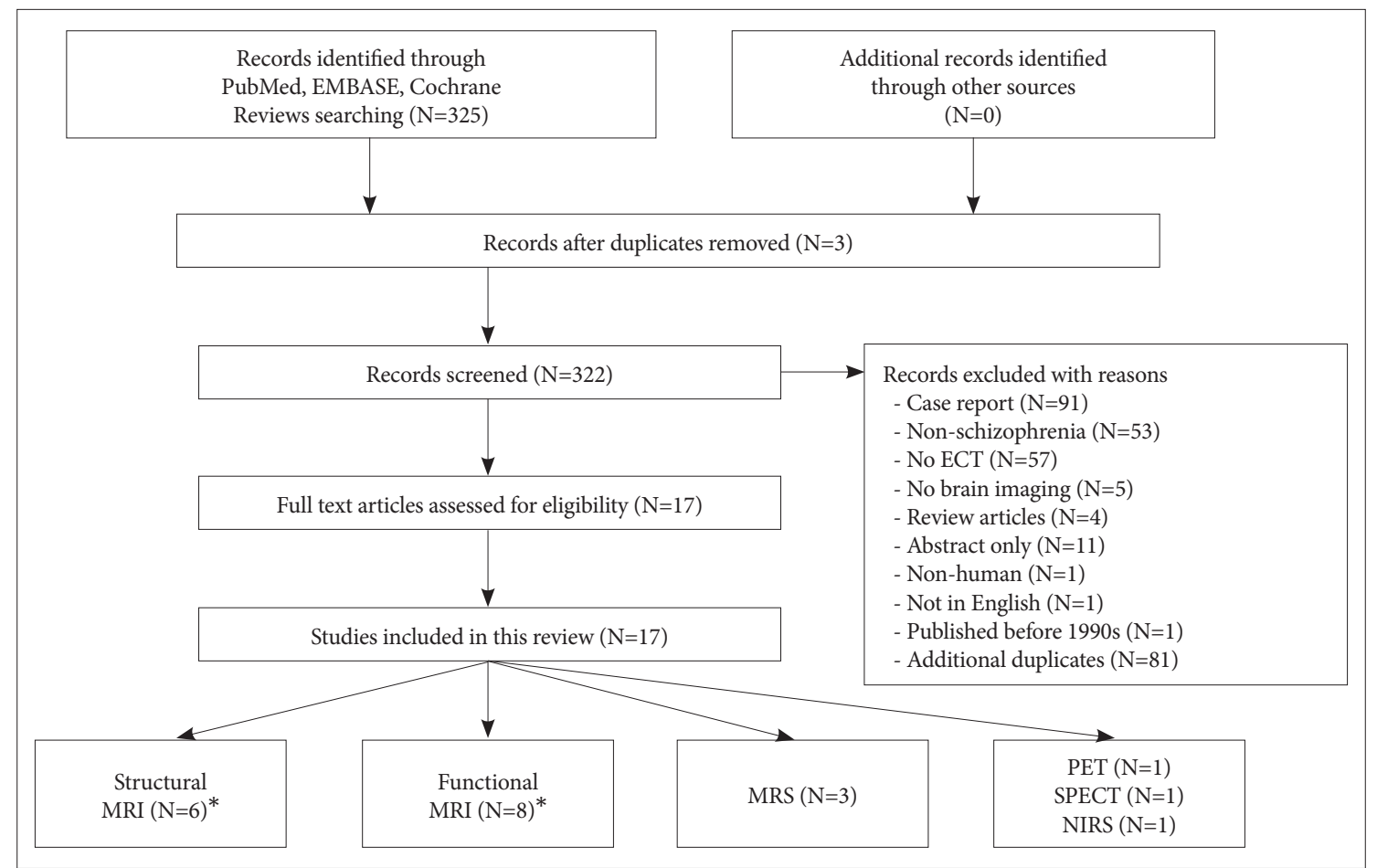

Figure 1. Flowchart of the literature review process. ${ }^{*}$ Three studies studied both structural and functional MRI changes after ECT, and thus was included in both structural and functional MRI categories. ECT: electroconvulsive therapy, MRI: magnetic resonance imaging, MRS: magnetic resonance spectroscopy, PET: positron emission tomography, SPECT: single positron emission computer tomography, NIRS: near-infrared spectroscopy.

Table 1. Cerebral blood flow in patients with schizophrenia receiving electroconvulsive therapy (ECT)

\begin{tabular}{|c|c|c|}
\hline & Baseline & After ECT \\
\hline Comparisons with MDD patients & No difference $(\text { SPECT) })^{37}$ & $\begin{array}{l}\uparrow \text { Rt. parietal and lt. and rt. temporal CBF (SPECT) }{ }^{37} \\
\uparrow \text { Lt. to rt. PFC ratio (NIRS) }\end{array}$ \\
\hline Comparisons with controls & $\begin{array}{l}\uparrow \text { Both temporal lobes and lt. } \\
\text { cerebellum }(\mathrm{PET})^{36}\end{array}$ & \\
\hline Within-group comparisons (baseline vs. after & & No difference $(\text { SPECT })^{37}$ \\
\hline ECT) in schizophrenia patients with ECT & & $\begin{array}{l}\downarrow \text { Lt. and rt. frontal, rt. temporal, and rt. } \\
\text { putamen } \mathrm{CBF}(\mathrm{PET})^{36}\end{array}$ \\
\hline
\end{tabular}

MDD: major depressive disorder, lt: left, rt: right, CBF: cerebral blood flow, SPECT: single positron emission computer tomography, PFC: pefrontal cortex, NIRS: near-infrared spectroscopy, PET: positron emission tomography

\section{Effect of ECT on brain metabolites}

Three studies examined the changes in brain metabolites using MRS (Table 2). Two independent studies ( $\mathrm{n}=34$ and $\mathrm{n}=$ 10) revealed changes of $\mathrm{N}$-acetyl-aspartate/creatinine (NAA/ Cr) ratio in the left PFC and the left thalamus. ${ }^{39,40}$ Another study showed an increase in medial prefrontal $\gamma$-aminobutyric acid (GABA) levels $(\mathrm{n}=14){ }^{41}$

\section{Effect of ECT on brain structures}

Six studies investigated structural changes after ECT in patients with schizophrenia. Table 3 demonstrates the findings of each study according to the method of analysis [wholebrain analysis or region-of-interest (ROI) analysis]. Notably, three datasets from three different study groups were ana- lyzed, and using different and complementary analysis methods, a total of six publications were included. This relatively small number of studies may be partially attributed to the relatively restricted use of ECT in some parts of the world, ${ }^{11}$ with varying treatment guidelines across nations, and a series of index ECT treatments require approximately one month to perform, which raises the issue of accessibility.

One group compared the effects of ECT on patients with schizophrenia ( $\mathrm{n}=9)$ and MDD $(\mathrm{n}=12)^{42,43}$ Based on the results of the whole-brain analysis, the authors found that the gray matter volume (GMV) in the medial temporal lobe (MTL) network and the left dorsolateral prefrontal cortex (DLPFC) increased in patients with schizophrenia after ECT. ${ }^{42}$ An additional complementary analysis of the temporal regions re- 
Table 2. Brain metabolites measured by magnetic resonance spectroscopy in patients with schizophrenia receiving electroconvulsive therapy (ECT)

\begin{tabular}{lll}
\hline & \multicolumn{1}{c}{ Baseline } & \multicolumn{1}{c}{ After ECT } \\
\hline Comparisons with controls & $\downarrow$ NAA/Cr ratios in the lt. PFC and lt. thalamus ${ }^{39}$ & \\
& $\downarrow$ Medial frontal GABA ${ }^{41}$ & \\
Comparisons with schizophrenia & & NAA/Cr ratios in the lt. PFC and lt. thalamus ${ }^{39}$ \\
patients who did not receive ECT & & NAA/Cr ratio in the lt. PFC ${ }^{39}$ \\
and underwent antipsychotic & & $\downarrow$ Cho/Cr ratios in the lt. PFC and lt. thalamus ${ }^{40}$ \\
treatment only & & \\
\hline
\end{tabular}

NAA/Cr: N-acetyl-aspartate/creatinine, lt: left, GABA: gamma-aminobutyrate, PFC: prefrontal cortex, Cho/Cr: choline/creatinine

vealed that increases in volumes of the hippocampus and insula were shared by patients with schizophrenia and MDD, but were restricted to the right side..$^{43}$ The post hoc withingroup analyses only revealed increased GMV in the right insula in the schizophrenia group.

Another group examined the effects of ECT on patients with schizophrenia (i.e., ECT+antipsychotic medication; $\mathrm{n}=21$ ) compared with schizophrenia patients who did not receive ECT and underwent antipsychotic treatment only $(\mathrm{n}=21){ }^{44-46}$ Whole-brain analyses showed increased GMVs in the bilateral parahippocampal gyri/hippocampi, right temporal pole/right superior temporal gyrus (STG), and right insula after ECT compared to baseline and the antipsychotics only group. ${ }^{44}$ Additional ROI analyses of the insula and hippocampus indicated increased GMVs in the bilateral dorsal anterior insula and posterior insula ${ }^{46}$ and bilaterally increased hippocampal volumes. ${ }^{45}$ An analysis stratified according to response status to ECT further showed that higher baseline volumes in the left hippocampus-amygdala transition area (HATA) were observed in responders. ${ }^{45}$

A separate study group reported that baseline GMVs in the temporal region, including the right insula, left middle temporal gyrus (MTG), and the right STG, along with the left inferior frontal gyrus (IFG), predicted ECT response. ${ }^{47}$

While the specific findings differed among studies, reviewed results overlap in findings of increased GMVs in the hippocampus (whole-brain analysis, $\mathrm{n}=2$; ROI analysis, $\mathrm{n}=2$ ) and the right insula (whole-brain analyses, $n=2$; ROI analysis, $n=2$ ).

\section{Effect of ECT on functional connectivity}

Eight studies from four different groups examined restingstate functional connectivity (rsFC) after ECT. Table 4 demonstrates the findings of each study according to the method of analysis (whole-brain analysis or ROI analysis). Findings common to these studies were changes in the rsFC of the default mode network (DMN), PFC, thalamus, hippocampus, and insula.

The first study group, which included schizophrenia $(\mathrm{n}=9)$ and $\operatorname{MDD}(\mathrm{n}=12)$ patients, performed a whole-brain analysis and observed increased rsFC of the medial prefrontal cortex (mPFC) within the DMN, between the executive network and the DMN, and between the executive network and salience network, along with decreased low-frequency oscillations in the striatal networks in patients with schizophrenia after ECT ${ }^{48}$ ROI analyses of the amygdala to other brain regions showed decreased rsFC between the right amygdala and various regions, including the mPFC, insula, and DLPFC. ${ }^{43}$

The second study group compared patients with schizophrenia treated with either ECT $(\mathrm{n}=21)$ or antipsychotics alone $(\mathrm{n}=21)$. Whole-brain analyses showed increased connectivity of the ventral medial prefrontal cortex (vmPFC) and dorsal medial prefrontal cortex (dmPFC) after ECT compared with the antipsychotics only group and controls. ${ }^{49}$ This group performed ROI analyses of the insular subfields, ${ }^{46}$ hippocampus, ${ }^{45}$ and thalamic subfields. ${ }^{50}$ While significant changes in various regions were found, responders to ECT exhibited increased rsFC of the hippocampus to the PFC and the hippocampus to the $\mathrm{DMN}^{45}$ and between the thalamus and regions of the temporal lobe and the cerebellum. ${ }^{50}$

The third group simulated electrical fields of ECT stimulation to select regions with large electrical fields, and compared the changes in those regions before and after ECT $(n=47) .{ }^{51}$ After ECT, rsFC between the right amygdala and left hippocampus was increased compared to baseline.

The fourth group observed altered rsFC within various networks, including the DMN and temporal lobe networks, in the ECT group compared with antipsychotics only group. ${ }^{52}$

The results from the aforementioned studies suggest rsFC of the DMN and associated regions ( $n=5), \operatorname{PFC}(n=4)$, thalamus $(n=2)$, hippocampus $(n=2)$ and insula $(n=2)$ were commonly altered after ECT.

\section{Associations with clinical improvement and ECT response status}

Findings of significant associations with clinical improvement and the status of the ECT response are listed in Supplementary Tables 2 and 3 (in the online-only Data Supplement), respectively. Four studies reported associations of clinical im- 


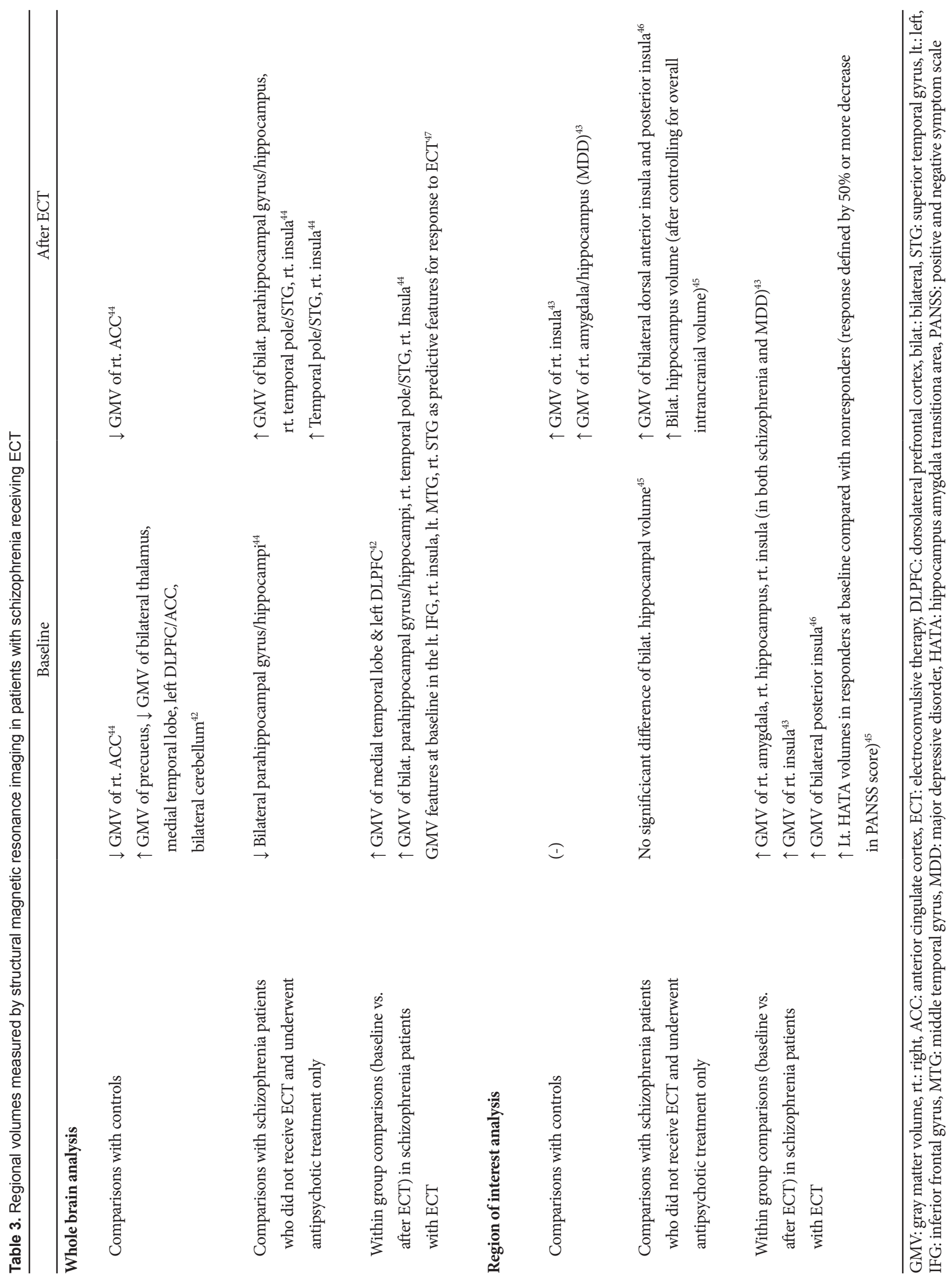


SY Moon et al.

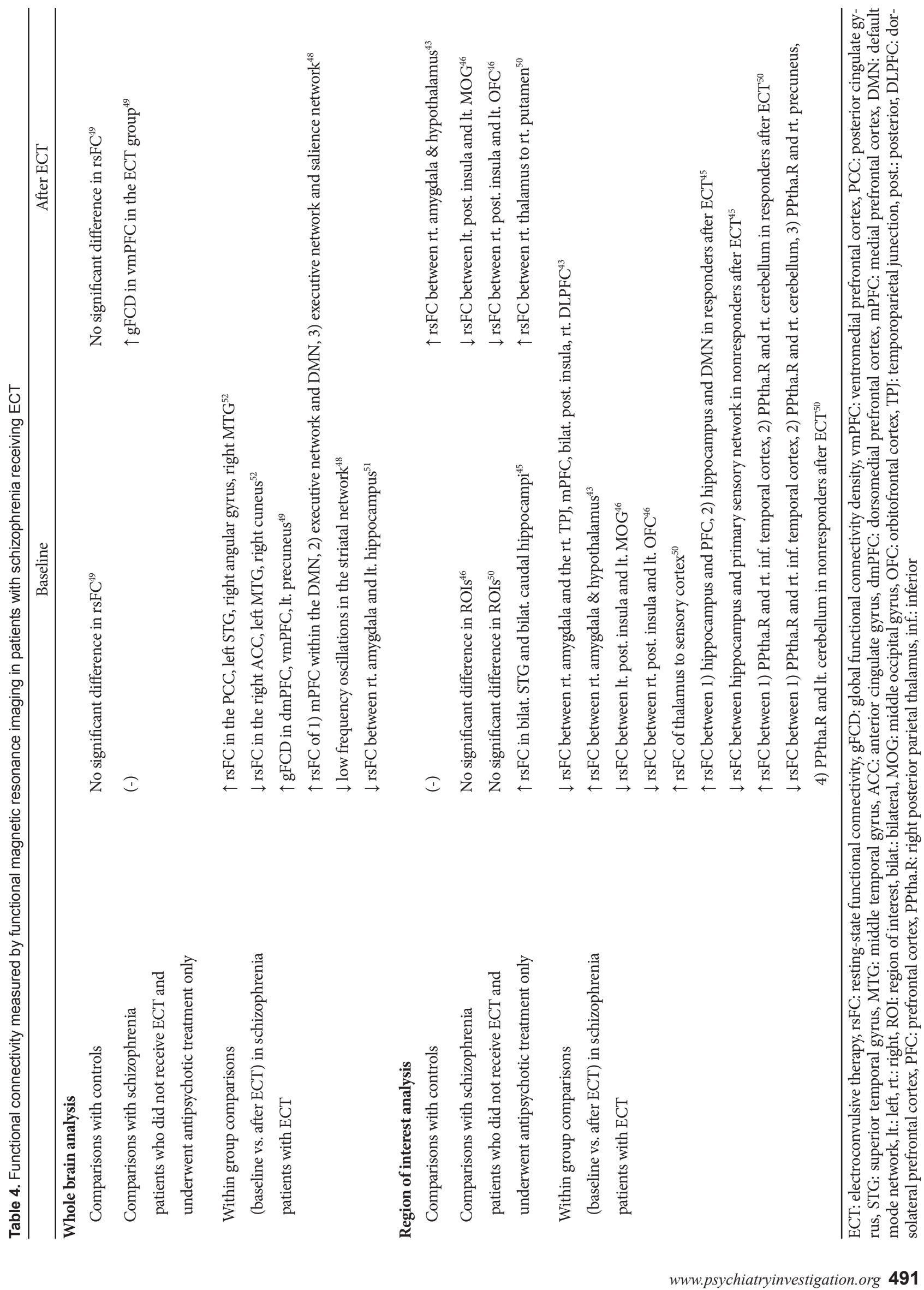


provement with changes in the hippocampus, ${ }^{44,45,51,52}$ two studies with changes in the insula, ${ }^{46,47}$ and one study with changes in the amygdalar region, respectively. ${ }^{51}$

\section{DISCUSSION}

This is the first systematic review to examine neural effects of ECT in patients with schizophrenia. A standardized literature search yielded 17 studies. The majority of recent studies $(\mathrm{n}=11)$ used MRI as the study modality, which examined morphometric $(\mathrm{n}=6)$ and functional $(\mathrm{n}=8)$ changes before and after ECT. An additional six studies investigated the effect of ECT on CBF $(n=3)$ and brain metabolites $(n=3)$. Overall, the reviewed publications considerably differed in both study designs and modalities, which precluded performing a metaanalysis.

\section{Cerebral blood flow changes after ECT}

Two studies compared changes in blood flow after ECT in patients with schizophrenia and patients with MDD and observed increased blood flow in patients with schizophrenia using SPECT and NIRS, respectively. ${ }^{37,38}$ Notably, studies of patients with MDD quite frequently report decreased blood flow in various regions following ECT sessions. ${ }^{53}$ Two other studies compared the data obtained from patients with schizophrenia before and after ECT, and one study did not report significant alterations (SPECT) ${ }^{37}$ while the other reported decreased blood flow after ECT (PET). ${ }^{36}$ Due to the small numbers and discrepancies in study modalities and findings, a solid conclusion concerning changes in blood flow after ECT is difficult to obtain.

\section{Changes in brain metabolites after ECT}

Other studies examined changes in brain metabolites using MRS; interestingly, two studies reported increased NAA/Cr ratios in the left PFC and the left thalamus. The NAA/Cr ratio is considered an indicator of neuronal integrity, ${ }^{54,55}$ which is known to decrease in conditions such as aging, brain tumors, and acute pathological processes, ${ }^{56}$ and these decreases are known to be reversible with treatment. ${ }^{55}$ Previous meta-analyses provided consistent evidence of decreased NAA/Cr ratios in the hippocampus, thalamus, frontal and temporal lobes of patients with schizophrenia, ${ }^{57}$ which, interestingly, were increased after antipsychotic treatment. ${ }^{58,59}$ Although these findings must be replicated in the future due to the small number of studies that utilized MRS, the increase in NAA/Cr ratios in the left PFC and the left thalamus, regions that are strongly implicated in the pathophysiological hypothesis of schizophrenia, ${ }^{60-63}$ may imply therapeutic neuronal 'reorganization' by ECT. These results from MRS, since they contain proper- ties of both structure and function, might serve integrative functions in explaining the effects of ECT when results from MRI studies are combined along with the proposed pathophysiological models of schizophrenia. Future studies examining NAA, GABA, and glutamate or glutamine in the regions highly implicated in schizophrenia and ECT (such as the frontal and temporal lobes, the hippocampus, and thalamostriatal regions), may help to further our understanding of the mechanism of action of ECT.

\section{Structural and functional changes after ECT}

Only four study groups independently examined the neural effects of ECT on patients with schizophrenia. Although fewer studies of patients with schizophrenia have been reported than studies of patients with depression, the results quite consistently indicate increases in the volumes of the hippocampus (structural, $n=4$ ) and insula (structural, $n=4$ ). Additionally, a comparison of patients with schizophrenia and MDD revealed common increases in the GMVs of the hippocampus, while the increases in right insular GMV were restricted to patients with schizophrenia. ${ }^{43} \mathrm{FC}$ studies presented more divergent results for different ROIs, while commonly identified alterations were located within the regions of the DMN (functional, $\mathrm{n}=5), \operatorname{PFC}(\mathrm{n}=4)$, thalamostriatal system $(\mathrm{n}=2)$, hippocampus $(\mathrm{n}=2)$, and insula $(\mathrm{n}=2)$. Moreover, among the features that were associated with symptom reductions were the hippocampus $(n=4)$, insula $(n=2)$, and amygdala $(n=1)$.

From the integrated findings of both structural and functional measures, we infer, albeit preliminarily, that the hippocampus (total, $\mathrm{n}=6$; symptom associations, $\mathrm{n}=4$ ) and insula (total, $\mathrm{n}=6$; symptom associations, $\mathrm{n}=2$ ) are the common regions of modulation in patients with schizophrenia after ECT.

\section{Insula}

The insula is located deep within the lateral sulcus of the human brain. The upper cortices that cover the insula are called the operculum, which are composed of parts of the adjacent frontal, temporal, and parietal cortices. The insula is involved in various functions, such as interoception, multimodal sensory processing, ${ }^{64}$ self-related processes, ${ }^{65-68}$ taste, ${ }^{69}$ social emotions, ${ }^{70-73}$ homeostasis $\mathrm{s}^{74,75}$ and auditory perception. ${ }^{76-78}$ This whole spectrum of functions can be served owing to the extensive viscerosensory inputs into the region and its anatomical position amidst three different cerebral lobes permitting dense reciprocal connections with the limbic, prefrontal, somatosensory, and temporal areas. ${ }^{79,80}$

The results from the current systematic review revealed increased GMVs in the right insula in all of the structural MRI studies that performed whole-brain analysis. While conclusion should not be drawn solely based on this result, the un- 
derlying implication of insular involvement in patients with schizophrenia suggests that the insula has some unique characteristics worth further discussion.

First, the insula is one of the most consistent regions showing structural alterations in patients with schizophrenia. An anecdotal meta-analysis that included studies performing wholebrain analyses showed that approximately $50 \%$ and $40 \%$ of studies (which ranks as 7th and 10th, respectively among all regions of interest) reported reduced volumes of left and right insular areas, respectively. ${ }^{81}$ Indeed, structural alterations of the insula are considered the most consistent findings in psychosis subjects. ${ }^{82}$ In a meta-analysis performed by Crow et al., ${ }^{82}$ the insula was the most commonly reported area showing volumetric reduction, followed by the thalamus and anterior cingulate cortex. Additionally, in terms of numbers, right insula was the most commonly reported altered area, while in terms of the size of volumetric reductions, left insular defects were more profound in patients with schizophrenia. ${ }^{82}$

Second, recent studies have demonstrated insular involvement in auditory sensory processing. Intracranial electroencephalography recordings found auditory responses of the posterior insula resembling the response in Heschl's gyrus, ${ }^{77}$ and a separate study implicated insular involvement in auditory deviance detection and the formation of mismatch negativity. ${ }^{78}$ Abnormal sensory gating and altered auditory evoked potentials are one of the key pathophysiological findings explaining the phenomena of hallucinations in schizophrenia patients. ${ }^{83}$ Since clinical observations have suggested that ECT is more effective in refractory positive symptoms than in negative domains, ${ }^{15}$ amelioration of clinical symptoms after ECT might be mediated in part by modulation of the insular cortex.

Third, although its implications in schizophrenia remain unclear, lateralized functions of the insula appear to be related to the control of the autonomic nervous system. Various studies ranging from intraoperative intracranial electrical stimulation to lesion studies have shown that the right insula is involved in the top-down control of sympathetic tone. ${ }^{75,84-86}$ While the findings have been somewhat inconsistent, various types of autonomic dysfunction, such as alterations in heart rate variability $(\mathrm{HRV})^{87,88}$ and decreased vagal tone ${ }^{89,90}$ have been reported in patients with schizophrenia. Autonomic responses and control during stressful situations constitute an important axis in the stress diathesis model of psychiatric illness, ${ }^{91-93}$ and insular modulation by ECT may exert some effects on the central control of sympathetic nervous system tone.

Finally, the self-related functions of insula share striking commonalities in terms of the main psychopathology of delusions and hallucinations in patients with schizophrenia. Among the wide range of insular dysfunctions demonstrated in patients with schizophrenia, ${ }^{94-100}$ some studies have report- ed a relationship between decreased right insular volumes and delusion severity ${ }^{94}$ or degree of insight. ${ }^{97}$ Delusion and hallucination are considered externalizing psychopathologies, which suggest impairment in the insular functions of interoception and self-attribution in patients with schizophrenia.

\section{Hippocampus}

The modulation of the hippocampus and its neurotrophic functions by ECT are very strongly supported by findings from animal models of electroconvulsive seizure (ECS) and patients with MDD. The increase in the hippocampal volume observed in patients with depression after ECT are not only a robust finding but also explains many aspects of depression, including its treatment and etiology. Concerning treatment, ECT is considered the most effective treatment modality in depression, including treatment-resistant cases. ${ }^{13,14}$ Approximately $50 \%$ to $60 \%$ of treatment-resistant depression patients will respond to ECT, ${ }^{13}$ and its efficacy is deemed to be five- to six-fold greater than antidepressant therapy. In addition, the chronic stress model of depression is closely associated with dysfunction of the hypothalamic-pituitary-adrenal (HPA) axis, ${ }^{101}$ as both early life and chronic stress are known to increase levels of the stress hormone cortisol and alter the functions of the HPA axis. ${ }^{102}$ Stress and HPA axis dysfunction are known to exert deleterious effect on hippocampus, including cell loss and volume reductions. ${ }^{103,104}$

Meanwhile, most researchers appreciate that schizophrenia is a 'dysconnection' syndrome among large distributed brain networks, ${ }^{105,106}$ rather than problem of a single faulty region. Notwithstanding, hippocampal hyperactivity is among the most consistent functional abnormalities observed in patients with schizophrenia. ${ }^{107-109}$ Postmortem studies have replicated reduced hippocampal volumes, ${ }^{110-112}$ which are also consistent with the current body of neuroimaging studies in patients with schizophrenia. ${ }^{113-116}$ In addition, while the number of neurons in the hippocampus is not significantly altered, reduction in inhibitory interneuron population of the hippocampus was demonstrated. ${ }^{107,108,117}$ Studies measuring blood flow and metabolism also replicated abnormalities in the hippocampus, and subsequent studies have found that these metabolic shifts are associated with a greater reduction in hippocampal volume with disease progression. ${ }^{109}$ Additionally, neuroimaging and animal studies have consistently reported reductions in the volumes of hippocampal subfields, with CA1 subfield showing the most robust changes. ${ }^{118,119}$

The dopamine hypothesis of schizophrenia was born based on the clinical effectiveness of antipsychotic agent chlorpromazine. $^{120-122}$ The modern mainstream pathophysiological hypothesis of schizophrenia focuses on abnormality of the glutamate system and impairment in interneuron functions. ${ }^{123-125}$ 
Hippocampal hyperactivity can be explained by both the dopamine and glutamate hypotheses. First, while optimal levels of dopamine can enhance memory and functions subserved by the hippocampus, higher levels of dopamine were demonstrated to exert deleterious effects. ${ }^{112,126,127}$ Neuronal connections between the ventral hippocampus and striatum are bidirectional and capable of modulating dopamine levels in the other structure. ${ }^{126,128,129}$ This means that the dysfunctionally increased dopamine levels in the striatum can induce high dopamine levels in hippocampal areas, and vice versa, which could lead to hippocampal hyperactivity. Second, the hippocampus is among one of the most implicated areas concerning the glutamate hypothesis, which includes NMDA receptor hypofunction and reduced inhibitory interneuron function. ${ }^{107}$ These interneuron abnormalities result not only in hyperactivity of the hippocampus but also in dysfunctional connectivity, which includes connections to the striatum and the frontal lobes. ${ }^{126,129,130}$

Moreover, with regard to the neurotrophic hypothesis of ECT, there is considerable evidence that neurogenesis functions are altered in schizophrenia. ${ }^{131-133}$ Animal models of psychosis have demonstrated decreased adult neurogenesis. ${ }^{134-136}$ However, animal models of schizophrenia are mainly induced by administering substances known to induce psychotic illness. ${ }^{129}$ While the psychotic state itself is indeed important in understanding the pathology of schizophrenia, it may not be sufficient since schizophrenia follows a more chronic and deteriorating clinical course, including negative symptoms and cognitive impairment. Tracing adult neurogenesis in vivo in patients is methodologically difficult due to technical issues, and thus surrogate markers are frequently used to measure adult neurogenesis, such as Nestin, Ki-67 and Musashi-1, which are expressed in proliferating cells. ${ }^{132,137-139}$ Of such markers, downregulation of Ki-67 has been observed in the dentate gyrus of patients with schizophrenia compared to controls. ${ }^{139}$ In addition, induced pluripotent stem cell studies have modeled deficient functioning of dentate gyrus granule cells in schizophrenia, marked by alterations in gene expression of adult neurogenesis and decreased functioning. ${ }^{140}$ Additionally, numerous genes implicated in schizophrenia, such as DISC1, Neuroregulin-1, Reelin, are associated with neurogenesis. ${ }^{132,141}$ Although the direct findings of dysfunctional adult neurogenesis in patients with schizophrenia await verification, these functions are presumed to be altered when the previous literature is considered.

\section{Specificity of the findings and implications}

While these results provide evidence of changes in the hippocampus and insula of patients with schizophrenia, several important issues must be considered. First, might these results arise from nonspecific changes induced by ECT, such as edema, inflammation, or reactive gliosis $?^{30}$ While this is a possibility that needs to be addressed more thoroughly in future studies, existing evidence from both animal and human studies suggests that the effects of ECT cannot be fully attributed to these nonspecific effects. While ECS in animal models potentiates a wide range of alterations covering various regions of the brain, the results of ECS studies demonstrate rather specific changes in the hippocampal region with marked alterations in neuroplastic functions. ${ }^{142}$ Additionally, in human studies, neuroimaging within 15 hours after ECT did not detect marked change in fluid shifts in the hippocampal region. ${ }^{30,143}$ Moreover, studies of patients with MDD that controlled for overall cerebral volumes to address these issues have also reported a significant increase in the volume of the hippocampus apart from such nonspecific effects. ${ }^{30,144-147}$

Second, are these changes common both to MDD and schizophrenia, or are they disease-specific? Previous attempts to explain the mechanisms underlying ECT have largely focused on the overall ECT effect, without differentiating between the two disorders. ${ }^{26}$ While conclusions based on the currently insufficient body of evidence may be preliminary, the hippocampus appears to be commonly modulated in both animals and human subjects (including both patients with MDD and schizophrenia), while comparisons of patients with MDD with patients with schizophrenia observed increased volumes in the right insula restricted to the schizophrenia group. ${ }^{43}$ However, this evidence is preliminary and needs replication, and current research is even more scarce in terms of what are the requisite conditions for ECT to be effective and the relationship between hippocampal modulation and symptom improvements.

An important difference in the application of ECT between patients with MDD and schizophrenia is that ECT results in a very high proportion of responses and remission among patients with MDD; ${ }^{148}$ however, approximately one-third of patients with schizophrenia do not adequately benefit from ECT treatment. ${ }^{19}$ Therefore, to clearly delineate schizophrenia-specific ECT treatment effects from the common effects of electrical stimulation, classifying patients into responders and nonresponders and comparing between the two groups might prove useful in future studies.

Furthermore, as the discovery of the effect of chlorpromazine preceded the development of the dopamine hypothesis, efforts to identify the mechanism of ECT may also help to improve our understanding of treatment-resistance in patients with schizophrenia. While treatment-resistance constitutes a serious mental health problem, the neurobiological underpinnings of TRS are at best only poorly understood due to the lack of consistent findings hitherto. ${ }^{149-151}$ Because it can now be ap- 
preciated, based on large volumes of previous animal and MDD research, that the effects of ECT are specific to some regions, establishing the neurobiological substrates of ECT and combining them with stratification based on treatment response may provide some valuable insights to understanding the neural underpinnings of TRS.

\section{Limitations}

There are several limitations of this review. First and most importantly, a small number of studies with divergent modalities precluded a systematic meta-analysis. Second, individually selected ROIs among various studies also made it difficult to infer which region or networks were most consistently modulated after ECT in schizophrenia patients. Third, as discussed above, hippocampal and insular modulation might be a common end result of ECT and may not define schizophrenia-specific alterations.

\section{CONCLUSIONS}

This systematic review presents preliminary evidence of hippocampal and insular modulation after ECT in patients with schizophrenia. This holds true for morphometry, FC, and symptom association measures. Other potential candidates include the DMN, PFC, and thalamostriatal system, as demonstrated by MRS and fMRI studies, and the amygdala, along with the hippocampus and insula, which were associated with symptom reductions. However, due to the small number of studies, replication is indispensable to generalize and elaborate our understanding of the neurobiological underpinnings of the ECT effect in schizophrenia patients.

We believe that ECT provides a unique window to the understanding and management of treatment-resistance in patients with schizophrenia. However, the effect and mechanism of action of ECT are most likely to be complex and possibly mediated by various structural, functional, and metabolic alterations. Dysfunctional neurotrophic factors in schizophrenia could partially be remedied by ECT, but other factors, including neurotransmitter changes, synaptic remodeling, and restoration of altered FC in other largely distributed networks, may also play an important role. To disentangle the mystifying mechanisms of ECT, augmentation of functional approaches (functional MRI, MRS, electroencephalography) with morphometric results might prove useful in future study designs.

\section{Supplementary Materials}

The online-only Data Supplement is available with this article at https://doi.org/10.30773/pi.2020.0438.

\section{Acknowledgments}

This research was supported by the Brain Research Program and the Ba- sic Science Research Program through the National Research Foundation of Korea (NRF), funded by the Ministry of Science, ICT \& Future Planning (Grant nos. 2017M3C7A1029610 and 2019R1C1C1002457).

\section{Conflicts of Interest}

The authors have no potential conflicts of interest to disclose.

\section{Author Contributions}

Conceptualization: Minah Kim, Jun Soo Kwon. Data curation: Sun-Young Moon, Minah Kim. Formal analysis: Sun-Young Moon, Minah Kim. Funding acquisition: Minah Kim, Jun Soo Kwon. Investigation: all authors. Methodology: Sun-Young Moon, Minah Kim. Project administration: Minah Kim. Resources: Minah Kim, Jun Soo Kwon. Supervision: Minah Kim. Validation: Minah Kim, Se Hyun Kim, Jun Soo Kwon. Writing—original draft: Sun-Young Moon. Writing_-review \& editing: Minah Kim, Se Hyun Kim, Jun Soo Kwon.

\section{ORCID iDs}

Sun-Young Moon

Minah Kim

Silvia Kyungjin Lho

Sanghoon Oh

Se Hyun Kim

Jun Soo Kwon

\section{REFERENCES}

1. James SL, Abate D, Abate KH, Abay SM, Abbafati C, Abbasi N. Global, regional, and national incidence, prevalence, and years lived with disability for 354 diseases and injuries for 195 countries and territories, 1990-2017: a systematic analysis for the Global Burden of Disease Study 2017. Lancet 2018;392:1789-1858.

2. Elkis H, Buckley PF. Treatment-resistant schizophrenia. Psychiatr Clin North Am 2016;39:239-265.

3. Essock SM, Hargreaves WA, Covell NH, Goethe J. Clozapine's effectiveness for patients in state hospitals: results from a randomized trial. Psychopharmacol Bull 1996;32:683-697.

4. Lindenmayer JP. Treatment refractory schizophrenia. Psychiatr Q 2000;71:373-384.

5. Kennedy JL, Altar CA, Taylor DL, Degtiar I, Hornberger JC. The social and economic burden of treatment-resistant schizophrenia. Int Clin Psychopharmacol 2014;29:63-76.

6. Nucifora FC Jr, Woznica E, Lee BJ, Cascella N, Sawa A. Treatment resistant schizophrenia: clinical, biological, and therapeutic perspectives. Neurobiol Dis 2018;131:104257.

7. Lieberman JA, Safferman AZ, Pollack S, Szymanski S, Johns C, Howard A, et al. Clinical effects of clozapine in chronic schizophrenia: response to treatment and predictors of outcome. Am J Psychiatry 1994; 151:1744-1752.

8. Fink M. Convulsive therapy: a review of the first 55 years. J Affect Disord 2001;63:1-15.

9. Chanpattana W, Andrade C. ECT for treatment-resistant schizophrenia. J ECT 2006;22:4-12.

10. Rosenquist PB, Youssef NA, Surya S, McCall WV. When all else fails. Psychiatr Clin North Am 2018;41:355-371.

11. Leiknes KA, Jarosh-von Schweder L, Høie B. Contemporary use and practice of electroconvulsive therapy worldwide. Brain Behav 2012; 2:283-344.

12. Sanghani SN, Petrides G, Kellner CH. Electroconvulsive therapy (ECT) in schizophrenia. Curr Opin Psychiatry 2018;31:213-222.

13. Loo C. ECT in the 21st century: optimizing treatment. J ECT 2010;26: 157.

14. Pagnin D, de Queiroz V, Pini S, Cassano GB. Efficacy of ECT in depression: a meta-analytic review. J ECT 2004;20:13-20.

15. Grover S, Sahoo S, Rabha A, Koirala R. ECT in schizophrenia: a re- 
view of the evidence. Acta Neuropsychiatr 2018;31:115-127.

16. Ali SA, Mathur N, Malhotra AK, Braga RJ. Electroconvulsive therapy and schizophrenia: a systematic review. Mol Neuropsychiatry 2019; 5:75-83.

17. Galletly C, Castle D, Dark F, Humberstone V, Jablensky A, Killackey E, et al. Royal Australian and New Zealand College of Psychiatrists clinical practice guidelines for the management of schizophrenia and related disorders. Aust N Z J Psychiatry 2016;50:410-472.

18. Grover S, Chakrabarti S, Kulhara P, Avasthi A. Clinical practice guidelines for management of schizophrenia. Indian J Psychiatry 2017;59 (Suppl 1):S19-S33.

19. Lally J, Tully J, Robertson D, Stubbs B, Gaughran F, MacCabe JH. Augmentation of clozapine with electroconvulsive therapy in treatment resistant schizophrenia: a systematic review and meta-analysis. Schizophr Res 2016;171:215-224.

20. Miller AL, Hall CS, Buchanan RW, Buckley PF, Chiles JA, Conley RR, et al. The texas medication algorithm project antipsychotic algorithm for schizophrenia. J Clin Psychiatry 2004;65:500-508.

21. Petrides G, Malur C, Braga RJ, Bailine SH, Schooler NR, Malhotra $\mathrm{AK}$, et al. Electroconvulsive therapy augmentation in clozapine-resistant schizophrenia: a prospective, randomized study. Am J Psychiatry 2015;172:52-58.

22. Pompili M, Lester D, Dominici G, Longo L, Marconi G, Forte A, et al. Indications for electroconvulsive treatment in schizophrenia: a systematic review. Schizophr Res 2013;146:1-9.

23. Zheng W, Cao XL, Ungvari GS, Xiang YQ, Guo T, Liu ZR, et al. Electroconvulsive therapy added to non-clozapine antipsychotic medication for treatment resistant schizophrenia: meta-analysis of randomized controlled trials. PLoS One 2016;11:e0156510.

24. Sinclair DJ, Zhao S, Qi F, Nyakyoma K, Kwong JS, Adams CE. Electroconvulsive therapy for treatment-resistant schizophrenia. Cochrane Database Syst Rev 2019;3:CD011847.

25. Teodorczuk A, Emmerson B, Robinson G. Revisiting the role of electroconvulsive therapy in schizophrenia: where are we now? Australas Psychiatry 2019;27:477-479.

26. Bolwig TG. How does electroconvulsive therapy work? Theories on its mechanism. Can J Psychiatry 2011;56:13-18.

27. Gbyl K, Videbech P. Electroconvulsive therapy increases brain volume in major depression: a systematic review and meta-analysis. Acta Psychiatr Scand 2018;138:180-195.

28. Nordanskog P, Dahlstrand U, Larsson MR, Larsson EM, Knutsson L, Johanson A. Increase in hippocampal volume after electroconvulsive therapy in patients with depression. J ECT 2010;26:62-67.

29. Takamiya A, Chung JK, Liang KC, Graff-Guerrero A, Mimura M, Kishimoto T. Effect of electroconvulsive therapy on hippocampal and amygdala volumes: systematic review and meta-analysis. $\mathrm{Br} \mathrm{J}$ Psychiatry 2018;212:19-26.

30. Wilkinson ST, Sanacora G, Bloch MH. Hippocampal volume changes following electroconvulsive therapy: a systematic review and metaanalysis. Biol Psychiatry Cogn Neurosci Neuroimaging 2017;2:327335.

31. Yrondi A, Péran P, Sauvaget A, Schmitt L, Arbus C. Structural-functional brain changes in depressed patients during and after electroconvulsive therapy. Acta Neuropsychiatr 2018;30:17-28.

32. Devanand DP, Dwork AJ, Hutchinson ER, Bolwig TG, Sackeim HA. Does ECT alter brain structure? Am J Psychiatry 1994;151:957-970.

33. Weiner RD. Does electroconvulsive therapy cause brain damage? Behav Brain Sci 2010;7:1-22.

34. Menken M, Safer J, Goldfarb C, Varga E. Multiple ECT: morphologic effects. Am J Psychiatry 1979;136:453.

35. Bouckaert F, Sienaert P, Obbels J, Dols A, Vandenbulcke M, Stek M, et al. ECT: its brain enabling effects: a review of electroconvulsive therapy-induced structural brain plasticity. J ECT 2014;30:143-151.

36. Uesugi H, Toyoda J, Iio M. Positron emission tomography and plasma biochemistry findings in schizophrenic patients before and after electroconvulsive therapy. Psychiatry Clin Neurosci 1995;49:131-135.

37. Escobar R, Rios A, Montoya ID, Lopera F, Ramos D, Carvajal C, et al. Clinical and cerebral blood flow changes in catatonic patients treated with ECT. J Psychosom Res 2000;49:423-429.

38. Fujita Y, Takebayashi M, Hisaoka K, Tsuchioka M, Morinobu S, Yamawaki S. Asymmetric alternation of the hemodynamic response at the prefrontal cortex in patients with schizophrenia during electroconvulsive therapy: a near-infrared spectroscopy study. Brain Res 2011; 1410:132-140.

39. Gan JL, Duan HF, Cheng ZX, Yang JM, Zhu XQ, Gao CY, et al. Neuroprotective effect of modified electroconvulsive therapy for schizophrenia. J Nerv Ment Dis 2017;205:480-486.

40. Lotfi M, Jahromi MG, Firoozabadi A, Jahromi LR. Effect of adjuvant electroconvulsive therapy compared to antipsychotic medication alone on the brain metabolites of patients with chronic schizophrenia: a proton magnetic resonance spectroscopy study. Iran J Psychiatry 2018;13:215-221.

41. Xia M, Wang J, Sheng J, Tang Y, Li C, Lim K, et al. Effect of electroconvulsive therapy on medial prefrontal $\gamma$-aminobutyric acid among schizophrenia patients. J ECT 2018;34:227-232.

42. Wolf RC, Nolte HM, Hirjak D, Hofer S, Seidl U, Depping MS, et al. Structural network changes in patients with major depression and schizophrenia treated with electroconvulsive therapy. Eur Neuropsychopharmacol 2016;26:1465-1474.

43. Thomann PA, Wolf RC, Nolte HM, Hirjak D, Hofer S, Seidl U, et al. Neuromodulation in response to electroconvulsive therapy in schizophrenia and major depression. Brain Stimul 2017;10:637-644.

44. Wang J, Tang Y, Curtin A, Xia M, Tang X, Zhao Y, et al. ECT-induced brain plasticity correlates with positive symptom improvement in schizophrenia by voxel-based morphometry analysis of grey matter. Brain Stimul 2019;12:319-328.

45. Jiang Y, Xu L, Li X, Tang Y, Wang P, Li C, et al. Common increased hippocampal volume but specific changes in functional connectivity in schizophrenia patients in remission and non-remission following electroconvulsive therapy: a preliminary study. Neuroimage Clin 2019;24:102081.

46. Jiang Y, Xia M, Li X, Tang Y, Li C, Huang H, et al. Insular changes induced by electroconvulsive therapy response to symptom improvements in schizophrenia. Prog Neuropsychopharmacol Biol Psychiatry 2019;89:254-262.

47. Gong J, Cui LB, Xi YB, Zhao YS, Yang XJ, Xu ZL, et al. Predicting response to electroconvulsive therapy combined with antipsychotics in schizophrenia using multi-parametric magnetic resonance imaging. Schizophr Res 2020;216:262-271.

48. Sambataro F, Thomann PA, Nolte HM, Hasenkamp JH, Hirjak D, Kubera KM, et al. Transdiagnostic modulation of brain networks by electroconvulsive therapy in schizophrenia and major depression. Eur Neuropsychopharmacol 2019;29:925-935.

49. Huang H, Jiang Y, Xia M, Tang Y, Zhang T, Cui H, et al. Increased resting-state global functional connectivity density of default mode network in schizophrenia subjects treated with electroconvulsive therapy. Schizophr Res 2018;197:192-199.

50. Wang J, Jiang Y, Tang Y, Xia M, Curtin A, Li J, et al. Altered functional connectivity of the thalamus induced by modified electroconvulsive therapy for schizophrenia. Schizophr Res 2020;218:209-218.

51. Yang X, Xu Z, Xi Y, Sun J, Liu P, Liu P, et al. Predicting responses to electroconvulsive therapy in schizophrenia patients undergoing antipsychotic treatment: baseline functional connectivity among regions with strong electric field distributions. Psychiatry Res 2020;299: 111059.

52. Li P, Jing RX, Zhao RJ, Ding ZB, Shi L, Sun HQ, et al. Electroconvulsive therapy-induced brain functional connectivity predicts therapeutic efficacy in patients with schizophrenia: a multivariate pattern recognition study. NPJ Schizophr 2017;3:21.

53. Abbott CC, Gallegos P, Rediske N, Lemke NT, Quinn DK. A review 
of longitudinal electroconvulsive therapy: neuroimaging investigations. J Geriatr Psychiatry Neurol 2014;27:33-46.

54. Moffett JR, Ross B, Arun P, Madhavarao CN, Namboodiri AMA. Nacetylaspartate in the CNS: from neurodiagnostics to neurobiology. Prog Neurobiol 2007;81:89-131.

55. Soares DP, Law M. Magnetic resonance spectroscopy of the brain: review of metabolites and clinical applications. Clin Radiol 2009;64: $12-21$.

56. Hollingworth W, Medina LS, Lenkinski RE, Shibata DK, Bernal B, Zurakowski D, et al. A systematic literature review of magnetic resonance spectroscopy for the characterization of brain tumors. AJNR Am J Neuroradiol 2006;27:1404-1411.

57. Steen RG, Hamer RM, Lieberman JA. Measurement of brain metabolites by $1 \mathrm{H}$ magnetic resonance spectroscopy in patients with schizophrenia: a systematic review and meta-analysis. Neuropsychopharmacology 2005;30:1949-1962.

58. Kubota M, Moriguchi S, Takahata K, Nakajima S, Horita N. Treatment effects on neurometabolite levels in schizophrenia: a systematic review and meta-analysis of proton magnetic resonance spectroscopy studies. Schizophr Res 2020;222:122-132.

59. Paslakis G, Träber F, Roberz J, Block W, Jessen F. N-acetyl-aspartate (NAA) as a correlate of pharmacological treatment in psychiatric disorders: a systematic review. Eur Neuropsychopharmacol 2014;24: 1659-1675.

60. Andreasen NC, O'Leary DS, Flaum M, Nopoulos P, Watkins GL, Ponto LLB, et al. Hypofrontality in schizophrenia: distributed dysfunctional circuits in neuroleptic-naïve patients. Lancet 1997;349: 1730-1734.

61. Andreasen NC, Paradiso S, O'Leary DS. "Cognitive dysmetria" as an integrative theory of schizophrenia: a dysfunction in cortical-subcortical-cerebellar circuitry? Schizophr Bull 1998;24:203-218.

62. Cho KIK, Kwak YB, Hwang WJ, Lee J, Kim M, Lee TY, et al. Microstructural changes in higher-order nuclei of the thalamus in patients with first-episode psychosis. Biol Psychiatry 2019;85:70-78.

63. Woodward ND, Karbasforoushan H, Heckers S. Thalamocortical dysconnectivity in schizophrenia. Am J Psychiatry 2012;169:10921099.

64. Bushara KO, Hanakawa T, Immisch I, Toma K, Kansaku K, Hallett M. Neural correlates of cross-modal binding. Nat Neurosci 2002;6:190195.

65. Craig ADB. How do you feel-now? The anterior insula and human awareness. Nat Rev Neurosci 2009;10:59-70.

66. Farrer C, Frith CD. Experiencing oneself vs another person as being the cause of an action: the neural correlates of the experience of agency. Neuroimage 2002;15:596-603.

67. Karnath HO, Baier B, Nägele T. Awareness of the functioning of one's own limbs mediated by the insular cortex? J Neurosci 2005;25:71347138.

68. Tsakiris M, Hesse MD, Boy C, Haggard P, Fink GR. Neural signatures of body ownership: a sensory network for bodily self-consciousness. Cereb Cortex 2006;17:2235-2244.

69. Pritchard TC, Macaluso DA, Eslinger PJ. Taste perception in patients with insular cortex lesions. Behav Neurosci 1999;113:663-671.

70. Phan KL, Wager T, Taylor SF, Liberzon I. Functional neuroanatomy of emotion: a meta-analysis of emotion activation studies in PET and fMRI. Neuroimage 2002;16:331-348.

71. Quarto T, Blasi G, Maddalena C, Viscanti G, Lanciano T, Soleti E, et al. Association between ability emotional intelligence and left insula during social judgment of facial emotions. PLoS One 2016;11:e0148621.

72. Sanfey AG. The neural basis of economic decision-making in the ultimatum game. Science 2003;300:1755-1758.

73. Singer T. The neuronal basis and ontogeny of empathy and mind reading: review of literature and implications for future research. Neurosci Biobehav Rev 2006;30:855-863.

74. Critchley HD. Neural mechanisms of autonomic, affective, and cog- nitive integration. J Comp Neurol 2005;493:154-166.

75. Oppenheimer SM, Gelb A, Girvin JP, Hachinski VC. Cardiovascular effects of human insular cortex stimulation. Neurology 1992;42:17271727.

76. Afif A, Minotti L, Kahane P, Hoffmann D. Anatomofunctional organization of the insular cortex: a study using intracerebral electrical stimulation in epileptic patients. Epilepsia 2010;51:2305-2315.

77. Blenkmann AO, Collavini S, Lubell J, Llorens A, Funderud I, Ivanovic J, et al. Auditory deviance detection in the human insula: an intracranial EEG study. Cortex 2019;121:189-200.

78. Zhang Y, Zhou W, Wang S, Zhou Q, Wang H, Zhang B, et al. The roles of subdivisions of human insula in emotion perception and auditory processing. Cereb Cortex 2019;29:517-528.

79. Augustine J. Circuitry and functional aspects of the insular lobe in primates including humans. Brain Res Rev 1996;22:229-244.

80. Duerden EG, Arsalidou M, Lee M, Taylor MJ. Lateralization of affective processing in the insula. Neuroimage 2013;78:159-175.

81. Honea R, Crow TJ, Passingham D, Mackay CE. Regional deficits in brain volume in schizophrenia: a meta-analysis of voxel-based morphometry studies. Am J Psychiatry 2005;162:2233-2245.

82. Crow TJ, Chance SA, Priddle TH, Radua J, James AC. Laterality interacts with sex across the schizophrenia/bipolarity continuum: an interpretation of meta-analyses of structural MRI. Psychiatry Res 2013; 210:1232-1244.

83. Smith DM, Grant B, Fisher DJ, Borracci G, Labelle A, Knott VJ. Auditory verbal hallucinations in schizophrenia correlate with P50 gating. Clin Neurophysiol 2013;124:1329-1335.

84. Chouchou F, Mauguière F, Vallayer O, Catenoix H, Isnard J, Montavont A, et al. How the insula speaks to the heart: cardiac responses to insular stimulation in humans. Hum Brain Mapp 2019;40:2611-2622.

85. Meyer S, Strittmatter M, Fischer C, Georg T, Schmitz B. Lateralization in autononic dysfunction in ischemic stroke involving the insular cortex. Neuroreport 2004;15:357-361.

86. Nagai M, Hoshide S, Kario K. The insular cortex and cardiovascular system: a new insight into the brain-heart axis. J Am Soc Hypertens 2010;4:174-182.

87. Castro MN, Vigo DE, Weidema H, Fahrer RD, Chu EM, de Achával $D$, et al. Heart rate variability response to mental arithmetic stress in patients with schizophrenia. Schizophr Res 2008;99:294-303.

88. Clamor A, Hartmann MM, Köther U, Otte C, Moritz S, Lincoln TM. Altered autonomic arousal in psychosis: an analysis of vulnerability and specificity. Schizophr Res 2014;154:73-78.

89. Chang JS, Yoo CS, Yi SH, Hong KH, Oh HS, Hwang JY, et al. Differential pattern of heart rate variability in patients with schizophrenia. Prog Neuropsychopharmacol Biol Psychiatry 2009;33:991-995.

90. Clamor A, Lincoln TM, Thayer JF, Koenig J. Resting vagal activity in schizophrenia: meta-analysis of heart rate variability as a potential endophenotype. Br J Psychiatry 2016;208:9-16.

91. Berntson GG, Cacioppo JT. Heart Rate Variability: Stress and Psychiatric Conditions. In: Malik M, Editor. Dynamic Electrocardiography. Bath: Blackwell Futura, 2004, p.57-64.

92. Thayer JF, Åhs F, Fredrikson M, Sollers JJ, Wager TD. A meta-analysis of heart rate variability and neuroimaging studies: implications for heart rate variability as a marker of stress and health. Neurosci Biobehav Rev 2012;36:747-756.

93. Vrijkotte TGM, van Doornen LJP, de Geus EJC. Effects of work stress on ambulatory blood pressure, heart rate, and heart rate variability. Hypertension 2000;35:880-886.

94. Cascella NG, Gerner GJ, Fieldstone SC, Sawa A, Schretlen DJ. The insula-claustrum region and delusions in schizophrenia. Schizophr Res 2011;133:77-81.

95. Jang DP, Kim JJ, Chung TS, An SK, Jung YC, Lee JK, et al. Shape deformation of the insula in schizophrenia. Neuroimage 2006;32:220227.

96. Linnman C, Coombs G 3rd, Goff DC, Holt DJ. Lack of insula reac- 
tivity to aversive stimuli in schizophrenia. Schizophr Res 2013;143: 150-157.

97. Palaniyappan L, Mallikarjun P, Joseph V, Liddle PF. Appreciating symptoms and deficits in schizophrenia: right posterior insula and poor insight. Prog Neuropsychopharmacol Biol Psychiatry 2011;35: 523-527.

98. Palaniyappan L, Simmonite M, White TP, Liddle EB, Liddle PF. Neural primacy of the salience processing system in schizophrenia. Neuron 2013;79:814-828

99. White TP, Joseph V, Francis ST, Liddle PF. Aberrant salience network (bilateral insula and anterior cingulate cortex) connectivity during information processing in schizophrenia. Schizophr Res 2010;123: 105-115.

100. Wylie KP, Tregellas JR. The role of the insula in schizophrenia. Schizophr Res 2010;123:93-104.

101. Gotlib IH, Joormann J, Minor KL, Hallmayer J. HPA axis reactivity: a mechanism underlying the associations among 5-HTTLPR, stress, and depression. Biol Psychiatry 2008;63:847-851.

102. Heim C, Newport DJ, Mletzko T, Miller AH, Nemeroff CB. The link between childhood trauma and depression: insights from HPA axis studies in humans. Psychoneuroendocrinology 2008;33:693-710.

103. Lee AL, Ogle WO, Sapolsky RM. Stress and depression: possible links to neuron death in the hippocampus. Bipolar Disord 2002;4:117-128.

104. Lucassen P, Heine V, Muller M, van der Beek E, Wiegant V, Ron De Kloet E, et al. Stress, depression and hippocampal apoptosis. CNS Neurol Disord Drug Targets 2006;5:531-546.

105. Friston K, Brown HR, Siemerkus J, Stephan KE. The dysconnection hypothesis (2016). Schizophr Res 2016;176:83-94.

106. Friston KJ, Frith CD. Schizophrenia: a disconnection syndrome. Clin Neurosci 1995;3:89-97.

107. Benes FM. Evidence for altered trisynaptic circuitry in schizophrenic hippocampus. Biol Psychiatry 1999;46:589-599.

108. Benes F, Berretta S. GABAergic interneurons implications for understanding schizophrenia and bipolar disorder. Neuropsychopharmacology 2001;25:1-27.

109. Heckers S, Konradi C. GABAergic mechanisms of hippocampal hyperactivity in schizophrenia. Schizophr Res 2015;167:4-11.

110. Bogerts B, Falkai P, Haupts M, Greve B, Ernst S, Tapernon-Franz U, et al. Post-mortem volume measurements of limbic system and basal ganglia structures in chronic schizophrenics. Schizophr Res 1990;3: 295-301.

111. Dwork AJ. Postmortem studies of the hippocampal formation in schizophrenia. Schizophr Bull 1997;23:385-402.

112. Heckers S, Heinsen H, Heinsen YC, Beckmann H. Limbic structures and lateral ventricle in schizophrenia. A quantitative postmortem study. Arch Gen Psychiatry 1990;47:1016-1022.

113. van Erp TG, Hibar DP, Rasmussen JM, Glahn DC, Pearlson GD, Andreassen OA, et al. Subcortical brain volume abnormalities in 2028 individuals with schizophrenia and 2540 healthy controls via the ENIGMA consortium. Mol Psychiatry 2016;21:547-553.

114. Okada N, Fukunaga M, Yamashita F, Koshiyama D, Yamamori H, Ohi $\mathrm{K}$, et al. Abnormal asymmetries in subcortical brain volume in schizophrenia. Mol Psychiatry 2016;21:1460-1466.

115. Koshiyama D, Fukunaga M, Okada N, Yamashita F, Yamamori H, Yasuda $\mathrm{Y}$, et al. Subcortical association with memory performance in schizophrenia: a structural magnetic resonance imaging study. Transl Psychiatry 2018;8:20.

116. Koshiyama D, Fukunaga M, Okada N, Yamashita F, Yamamori H, Yasuda $\mathrm{Y}$, et al. Role of subcortical structures on cognitive and social function in schizophrenia. Sci Rep 2018;8:1183.

117. Benes FM, Kwok EW, Vincent SL, Todtenkopf MS. A reduction of nonpyramidal cells in sector CA2 of schizophrenics and manic depressives. Biol Psychiatry 1998;44:88-97.

118. Adriano F, Caltagirone C, Spalletta G. Hippocampal volume reduction in first-episode and chronic schizophrenia. Neuroscientist 2012;
18:180-200.

119. Lieberman JA, Girgis RR, Brucato G, Moore H, Provenzano F, Kegeles $\mathrm{L}$, et al. Hippocampal dysfunction in the pathophysiology of schizophrenia: a selective review and hypothesis for early detection and intervention. Mol Psychiatry 2018;23:1764-1772.

120. Carlsson A. The current status of the dopamine hypothesis of schizophrenia. Neuropsychopharmacology 1988;1:179-186.

121. Howes OD, Kapur S. The dopamine hypothesis of schizophrenia: version III--the final common pathway. Schizophr Bull 2009;35:549-562.

122. Meltzer HY, Stahl SM. The dopamine hypothesis of schizophrenia: a review. Schizophr Bull 1976;2:19-76.

123. Hu W, MacDonald ML, Elswick DE, Sweet RA. The glutamate hypothesis of schizophrenia: evidence from human brain tissue studies. Ann N Y Acad Sci 2015;1338:38-57.

124. Moghaddam B, Javitt D. From revolution to evolution: the glutamate hypothesis of schizophrenia and its implication for treatment. Neuropsychopharmacology 2012;37:4-15.

125. Tamminga CA. Schizophrenia and glutamatergic transmission. Crit Rev Neurobiol 1998;12:21-36.

126. Grace AA. Dopamine system dysregulation by the hippocampus: implications for the pathophysiology and treatment of schizophrenia. Neuropharmacology 2012;62:1342-1348.

127. Lodge DJ, Grace AA. Hippocampal dysregulation of dopamine system function and the pathophysiology of schizophrenia. Trends Pharmacol Sci 2011;32:507-513.

128. Floresco SB, Todd CL, Grace AA. Glutamatergic afferents from the hippocampus to the nucleus accumbens regulate activity of ventral tegmental area dopamine neurons. J Neurosci 2001;21:4915-4922.

129. Grace AA. Ventral hippocampus, interneurons, and schizophrenia. Curr Dir Psychol Sci 2010;19:232-237.

130. Lodge DJ, Grace AA. Aberrant hippocampal activity underlies the dopamine dysregulation in an animal model of schizophrenia. J Neurosci 2007;27:11424-11430.

131. Reif A, Schmitt A, Fritzen S, Lesch KP. Neurogenesis and schizophrenia: dividing neurons in a divided mind? Eur Arch Psychiatry Clin Neurosci 2007;257:290-299.

132. Toro C, Deakin J. Adult neurogenesis and schizophrenia: a window on abnormal early brain development? Schizophr Res 2007;90:1-14.

133. Weissleder C, North HF, Shannon Weickert C. Important unanswered questions about adult neurogenesis in schizophrenia. Curr Opin Psychiatry 2019;32:170-178.

134. Duan X, Chang JH, Ge S, Faulkner RL, Kim JY, Kitabatake Y, et al. Disrupted-in-schizophrenia 1 regulates integration of newly generated neurons in the adult brain. Cell 2007;130:1146-1158.

135. Ouchi Y, Banno Y, Shimizu Y, Ando S, Hasegawa H, Adachi K, et al. Reduced adult hippocampal neurogenesis and working memory deficits in the Dgcr8-deficient mouse model of 22q11.2 deletion-associated schizophrenia can be rescued by IGF2. J Neurosci 2013;33:94089419.

136. Wolf SA, Melnik A, Kempermann G. Physical exercise increases adult neurogenesis and telomerase activity, and improves behavioral deficits in a mouse model of schizophrenia. Brain Behav Immun 2011;25: 971-980.

137. Blümcke I, Schewe JC, Normann S, Brüstle O, Schramm J, Elger CE, et al. Increase of nestin-immunoreactive neural precursor cells in the dentate gyrus of pediatric patients with early-onset temporal lobe epilepsy. Hippocampus 2001;11:311-321.

138. Crespel A, Rigau V, Coubes P, Rousset MC, de Bock F, Okano H, et al. Increased number of neural progenitors in human temporal lobe epilepsy. Neurobiol Dis 2005;19:436-450.

139. Reif A, Fritzen S, Finger M, Strobel A, Lauer M, Schmitt A, et al. Neural stem cell proliferation is decreased in schizophrenia, but not in depression. Mol Psychiatry 2006;11:514-522.

140. Yu DX, Di Giorgio FP, Yao J, Marchetto MC, Brennand K, Wright R, et al. Modeling hippocampal neurogenesis using human pluripotent 
stem cells. Stem Cell Rep 2014;2:295-310.

141. Bobilev AM, Perez JM, Tamminga CA. Molecular alterations in the medial temporal lobe in schizophrenia. Schizophr Res 2020;217:7185.

142. Altar CA, Laeng P, Jurata LW, Brockman JA, Lemire A, Bullard J, et al. Electroconvulsive seizures regulate gene expression of distinct neurotrophic signaling pathways. J Neurosci 2004;24:2667-2677.

143. Szabo K, Hirsch JG, Krause M, Ende G, Henn FA, Sartorius A, et al. Diffusion weighted MRI in the early phase after electroconvulsive therapy. Neurol Res 2007;29:256-259.

144. Abbott CC, Jones T, Lemke NT, Gallegos P, McClintock SM, Mayer AR, et al. Hippocampal structural and functional changes associated with electroconvulsive therapy response. Transl Psychiatry 2014;4:e483.

145. Joshi SH, Espinoza RT, Pirnia T, Shi J, Wang Y, Ayers B, et al. Structural plasticity of the hippocampus and amygdala induced by electroconvulsive therapy in major depression. Biol Psychiatry 2016;79:282-292.

146. Ota M, Noda T, Sato N, Okazaki M, Ishikawa M, Hattori K, et al. Effect of electroconvulsive therapy on gray matter volume in major de- pressive disorder. J Affect Disord 2015;186:186-191.

147. Tendolkar I, van Beek M, van Oostrom I, Mulder M, Janzing J, Voshaar $\mathrm{RO}$, et al. Electroconvulsive therapy increases hippocampal and amygdala volume in therapy refractory depression: a longitudinal pilot study. Psychiatry Res 2013;214:197-203.

148. Greenberg RM, Kellner CH. Electroconvulsive therapy: a selected review. Am J Geriatr Psychiatry 2005;13:268-281.

149. Gillespie AL, Samanaite R, Mill J, Egerton A, MacCabe JH. Is treatment-resistant schizophrenia categorically distinct from treatmentresponsive schizophrenia? A systematic review. BMC Psychiatry 2017; 17:12.

150. Mouchlianitis E, McCutcheon R, Howes OD. Brain-imaging studies of treatment-resistant schizophrenia: a systematic review. Lancet Psychiatry 2016;3:451-463.

151. Nakajima S, Takeuchi H, Plitman E, Fervaha G, Gerretsen P, Caravaggio F, et al. Neuroimaging findings in treatment-resistant schizophrenia: a systematic review: Lack of neuroimaging correlates of treatmentresistant schizophrenia. Schizophr Res 2015;164:164-175. 


\section{SUPPLEMENTARY MATERIAL}

\section{Supplementary methods}

PubMed, EMBASE, and the Cochrane Reviews were searched for publications with the following query: ("ECT" or "Electroconvulsive therapy" or "EST" or "Electroshock therapy" or "Electrotherapy") AND ("Schizophrenia" or "Schizo-" or "Schizoaffective" or "Psychosis" or "Psychotic") AND ("MRI" or "Magnetic resonance image" or "Magnetic resonance imaging" or "MRS" or "Magnetic resonance spectroscopy" or "Spectroscopy" or "PET" or "Positron emission tomography" or "BOLD” or "Blood oxygen level-dependent" or "Blood flow" or "SPECT" or "Single photon emission computed tomography" or "brain imag*" or "neuroimag*" or "DTI" or "Diffusion tensor" or "NIRS" or "Near infrared spectroscopy"). Two researchers (S.M. and M.K.) independently carried out literature search procedures.

We intended to include articles that 1) included patients with schizophrenia, 2) studied the effects of electroconvulsive therapy (ECT), 3) utilized at least one neuroimaging modality, and 4) were written in English. The exclusion criteria were as follows: 1) studies that did not involve patients with schizophrenia, 2) studies that did not implement ECT, 3) studies that did not use any brain imaging methods, 4) publications that were case reports or case series, 5) publications that were review articles, 6) studies involving nonhuman subjects, 6) articles published in languages other than English, 7) articles for which only the abstract was available, 8) publications that were errata, 9) duplicate publications, and 10) studies published before 1990, which were retrieved during the systematic review process.

\section{Supplementary results}

\section{Effects of ECT on CBF}

Of the three studies included in this review, each study utilized different modalities and the study/control groups varied (two studies compared patients with schizophrenia and patients with depression, and one study compared patients with schizophrenia with controls).

A PET study compared the effect of bifrontal ECT on patients with schizophrenia $(\mathrm{n}=5)$ and controls $(\mathrm{n}=6) .{ }^{1}$ Following ECT, the CBF in the bilateral frontal lobes, right temporal lobe, and right putamen were decreased in the schizophrenia group.

A study using SPECT evaluated the effect of bifrontotemporal ECT on patients with catatonic schizophrenia $(n=5)$ and catatonic depression $(n=5) .{ }^{2}$ Prior to ECT, the SPECT results did not differentiate between patients with schizophrenia and MDD with catatonia. One week after ECT completion, right parietal and bilateral temporal CBF was increased in patients with schizophrenia compared with patients with MDD. However, within-group comparisons including only the patients with schizophrenia did not show alterations compared to the baseline values.

The third study used NIRS to measure dynamic changes in blood flow prior to, during, and after each session of bifrontotemporal ECT in patients with schizophrenia $(\mathrm{n}=11)$ and MDD $(\mathrm{n}=10){ }^{3}$ After ECT, the schizophrenia group showed an increased blood flow ratio of the left prefrontal cortex (PFC) to the right PFC, which was an asymmetry that was not observed in the MDD group. The authors noted a negative correlation of the asymmetry index of the left to right PFC with the duration of illness in the schizophrenia group.

\section{Effects of ECT on brain metabolites}

Three studies utilized MRS to measure changes in brain metabolites after ECT in patients with schizophrenia.

Gan et al. ${ }^{4}$ used randomization to assign patients with schizophrenia $(n=68)$ into either ECT or antipsychotic treatment only groups and compared them with controls $(n=34)$. At baseline, the NAA/Cr ratio was lower in the left PFC and the left thalamus of the schizophrenia group than in the control group. After ECT, the NAA/Cr ratio in the left PFC and the left thalamus significantly increased, a change that was not observed in the antipsychotic treatment group. The left PFC and left thalamic NAA/Cr ratios also displayed significant associations with the demographic and clinical characteristics of the study group undergoing ECT treatment. Changes in the NAA/Cr ratio in the left PFC positively correlated with the age at onset ( $r=0.44)$, percent reduction in the Positive and Negative Syndrome Scale (PANSS) score ( $\mathrm{r}=0.41)$, baseline PANSS total score ( $\mathrm{r}=0.37)$, and ECT stimulus intensity $(\mathrm{r}=0.35)$, and negatively correlated with the duration of illness ( $\mathrm{r}=-0.41)$. Changes in the NAA/Cr ratio in the left thalamus positively correlated with the age at onset $(\mathrm{r}=0.33)$ and negatively correlated with the duration of illness $(\mathrm{r}=-0.35)$. The authors suggested that an older age at onset and a shorter duration of illness will result in a greater increase in the NAA/Cr ratio in the left PFC and left thalamus after treatment with ECT. 
Another study compared changes in metabolites in the prefrontal and thalamic regions of patients with schizophrenia after ECT $(\mathrm{n}=10)$ with patients undergoing antipsychotic treatment alone $(\mathrm{n}=10){ }^{5}$ After treatment, patients who received ECT presented a significantly higher $\mathrm{NAA} / \mathrm{Cr}$ ratio in the left prefrontal cortex than patients taking antipsychotic medications alone, and the choline/creatinine ratios in the left prefrontal and left thalamus were lower in the ECT group than in the antipsychotics alone group.

The third study measured medial prefrontal $\gamma$-aminobutyric acid (GABA) levels after ECT. ${ }^{6}$ Patients with schizophrenia either received ECT $(n=14)$ or antipsychotic treatment alone $(n=17)$, and controls $(n=19)$ were included. Medial prefrontal GABA levels did not significantly differ among the three groups, but when the group of patients with schizophrenia was analyzed as a whole, their GABA levels were lower than the control group. According to the post hoc analysis, GABA levels in the medial prefrontal cortex were increased in the ECT group, but not in the antipsychotics group.

\section{Effects of ECT on brain structures}

Six publications from three different groups were included in this review.

The first group examined the effect of ECT on patients with schizophrenia $(n=9)$, patients with MDD ( $n=12)$, and matched controls $(\mathrm{n}=21) .{ }^{7.8}$ Patient groups received right unilateral ECT. The whole-brain analysis yielded an increase in the GMV in the MTL network and the left DLPFC after ECT. ${ }^{8}$ The increases in the volume of the left DLPFC and changes in the total PANSS score showed a significant correlation $(\mathrm{r}=-0.70)$.

The results using a different analysis approach, i.e., the selection of an ROI within the same dataset, found increased GMVs in the right amygdala, the anterior part of the right hippocampus, and the right insula in the group of patients with schizophrenia and MDD combined after ECT. These changes did not correlate with symptom severity, but the baseline GMV in the amygdala displayed an inverse correlation with the post-ECT increases in GMV in this region ( $r=-0.75)$. Post hoc analyses revealed a significant within-group change in the right insula of patients with schizophrenia, while the results were not significant for patients with MDD. The post-ECT GMV values of the right amygdala/hippocampus region were greater in the MDD group and greater values were observed in the right insula in the schizophrenia group than the control values.

The second study group explored the effect of ECT on groups of patients with schizophrenia undergoing ECT ( $\mathrm{n}=21)$, patients with schizophrenia treated with antipsychotics alone $(n=21)$, and controls $(n=22) \cdot{ }^{9-11}$ Using the whole-brain analysis method, the results showed significant differences between the three groups in the 1) left parahippocampal gyrus/hippocampus, 2) right parahippocampal gyrus/hippocampus, 3) right temporal pole and mid/superior temporal gyrus, and 4) right insula. ${ }^{11}$ The post hoc analysis further revealed increased volumes of all four aforementioned regions in the ECT group compared to baseline, and the antipsychotics group actually presented decreased volumes compared to the pretreatment evaluation. Of the four regions, the increase in the volume of the right parahippocampal gyrus/hippocampus was associated with a reduction in score for the positive subscale of the PANSS ( $\mathrm{r}=0.574)$.

Complementary analyses by the same group used ROI analyses to explore the effect of ECT on regions of the insula ${ }^{9}$ and the hippocampus. ${ }^{10}$ Regarding the insula, the GMV in the posterior insula was increased in patients with schizophrenia after ECT sessions compared to baseline. ${ }^{9}$ These volumetric changes were also associated with changes in symptoms: increased GMV in the right posterior insula correlated with a reduced score for the PANSS positive scale $(\rho=0.667)$, reduced total PANSS score $(\rho=0.556)$, and reduced score for the PANSS general psychopathology scale $(\rho=0.634)$. With the selection of the hippocampus as the ROI, bilateral hippocampal volumes in the ECT group were increased after treatment compared to the antipsychotics only group (the overall intracranial volumes were adjusted).$^{10}$ Furthermore, the authors categorized the ECT group into treatment responders $(\mathrm{n}=10)$ and nonresponders $(\mathrm{n}=11)$ using a $50 \%$ or more reduction in the total PANSS score as the response criteria. While increased hippocampal volumes were observed in both responders and nonresponders, volumes in the left hippocampusamygdala transition area were larger in responders at baseline than in nonresponders.

The third study group used multiparametric markers of MRI to predict the effects of ECT measured by percent reductions in PANSS scores. ${ }^{12}$ The authors integrated baseline GM features and white matter features (WM), which used T1-weighted and diffusion-weighted imaging modalities, respectively. The selection of GM and WM tract ROIs was performed by simulating the electrical fields used during ECT stimulation by modeling, and those regions with an electrical field strength greater than $35 \mathrm{~V} / \mathrm{m}$ were included (23 GM ROIs and $37 \mathrm{WM}$ tracts). Models were tested to predict differences in PANSS scores before and after ECT, and the model with the highest performance was shown to include both GM and WM baseline features. The important GM features were the left inferior frontal gyrus (IFG), right insula, left middle temporal gyrus (MTG), and right superior temporal gyrus (STG), and the important WM tracts were between the left calcarine-left superior temporal pole, right lingual-right inferior temporal gyrus (ITG), left middle occipital gyrus (MOG)-left ITG, right MTG-right ITG, and right IFG-right insula. The selected 
model showed a performance with a root mean square error (RMSE) of 15.183 and a correlation efficient of 0.671 (RMSE $=0$ and $\mathrm{r}=1$ represent the most accurate predictive model). Furthermore, this model was tested in a separate group of patients with schizophrenia undergoing ECT ( $\mathrm{n}=15)$, which showed an RMSE value of 14.980 and a correlation coefficient of 0.777 .

\section{Effects of ECT on functional connectivity}

Eight studies from four different study groups were included in this review.

Thomann et al., ${ }^{7}$ whose study included both patients with schizophrenia and patients along with controls, found that right unilateral ECT appeared to alter the functional connectivity between the right amygdala and the ipsilateral cortical brain regions. The authors performed an ROI analysis with amygdala, hippocampus, and insula as seeds to assess the connectivity with the other brain regions. Reduced rsFC was observed between the right amygdala and the right temporo-parietal junction (TPJ), right medial prefrontal cortex (mPFC), bilateral posterior insula, and right DLPFC in both patient groups after ECT. In contrast, an increase in rsFC was observed between the right amygdala and hypothalamus, which follows the ventral amygdalofugal pathway, and these increases inversely correlated with the baseline connectivity strength in the same region $(r=-0.85)$. These changes were not associated with any symptom improvements in either group. Post hoc analyses only yielded significant increases in rsFC between the right amygdala and hypothalamus in the schizophrenia group after the course of ECT, which also did not correlate with symptom changes.

The same study group published another study using the whole-brain method. ${ }^{13}$ In patients with schizophrenia, the rsFC of the 1) $\mathrm{mPFC}$ within the DMN, 2) executive network and the DMN, and 3) executive networks and the salience network was increased after ECT completion. Additionally, ECT reduced low-frequency oscillations in the striatal network in patients with schizophrenia.

Another study group examined changes in functional connectivity after ECT and compared the ECT schizophrenia group, antipsychotic-only schizophrenia group, and controls. First, Huang et al. ${ }^{14}$ investigated the effect of ECT on the global functional connectivity density (gFCD) using resting-state functional magnetic resonance imaging (fMRI). The signal measured by gFCD construes rsFC for a particular voxel, with a higher signal density implying that the region serves as a functional hub. Significant differences were observed in the dorsal medial prefrontal cortex (dmPFC) and the ventral medial prefrontal cortex (vmPFC) among the ECT, antipsychotics only, and control groups. Within-group post hoc analyses showed increased gFCD in the dmPFC, vmPFC and left precuneus of the ECT group after the ECT course compared to the baseline.

Jiang et al. ${ }^{9}$ investigated the effect of ECT on changes in functional connectivity involving the insula. Significant group differences were observed among the ECT, antipsychotics only, and control groups between the left posterior insula and left MOG and between the right posterior insula and left orbitofrontal cortex (OFC). Post hoc analyses further revealed decreases in the functional connectivity strength between the left posterior insula and left MOG, as well as between the right posterior insula and left OFC, in the ECT group after ECT compared to the baseline, whereas the antipsychotics only group did not show significant changes. Interestingly, before the treatments, the ECT group presented slightly higher measures of functional connectivity (FC) in the aforementioned regions than the antipsychotics only group, but the ECT group exhibited substantially lower FC than the antipsychotics group after treatment. Additionally, FC between the left posterior insula and left MOG was significantly correlated with reduced scores on the PANSS general symptom scale $(\rho=0.587)$ and PANSS negative symptom scale $(\rho=0.560)$, and FC between the right posterior insula and left OFC was associated with reductions in the scores of the PANSS negative symptom scale $(\rho=0.460)$.

Jiang et al..$^{10}$ also explored FC between the hippocampus and other brain regions after ECT. Of the 21 patients who had undergone ECT treatment, 10 were classified as responders and 11 as nonresponders (a response was defined as a greater than $50 \%$ reduction in the total PANSS score). In responders, rsFC between the hippocampus and prefrontal cortex and between the hippocampus and DMN were increased after ECT compared to baseline. In nonresponders, post-ECT measures showed decreased rsFC between the hippocampus and primary sensory network compared to baseline.

In another complementary analysis performed by the same group, Wang et al. ${ }^{15}$ studied the effect of ECT on rsFC of the thalamic subfields. The authors compared the ECT plus antipsychotics group $(n=21)$ with the antipsychotics only group $(n=21)$. FC between the right thalamus and right putamen was increased in the ECT group compared to FC in the antipsychotics only group after treatment. Additionally, in the ECT group, FC of the thalamus to the sensory cortex was increased after treatment, while the antipsychotics group showed a decrease. When treatment responses were considered, responders to ECT showed increased FC between the right posterior parietal thalamus (PPthaR) and right inferior temporal cortex and between the PPthaR and right cerebellum, while FC in the same regions decreased in ECT nonresponders. The results of the studies that classified patients as responders and nonresponders are summarized in Supplementary Table 3 (in the online-only Data Supplement). 
The third study group utilized rsFC to predict ECT responses in patients with schizophrenia $(n=47) .{ }^{16}$ This same group used baseline GM and WM features to predict reductions in PANSS scores. ${ }^{12}$ The authors first simulated the hypothetical electric field distribution of the human head when an ECT stimulus was administered. Following this simulation, 23 ROIs presented high levels of electrical strength (i.e., greater than $35 \mathrm{~V} / \mathrm{m}$ ). Using these ROIs and the baseline FC of patients, they attempt to determine the best fitted model that would predict reductions in PANSS scores after ECT. A model with the most predictive values included 10 ROIs that would best predict the ECT response. Of these 10 ROIs, the actual data from study subjects showed a significant decrease in FC between the right amygdala and left hippocampus. The authors concluded that their model incorporating baseline FC would subsequently assist clinicians in determining individualized therapy and predict the ECT response at the individual level (Supplementary Table 2 in the online-only Data Supplement).

A separate research group built a classification model using $\mathrm{rsFC}$ to differentiate between patients with schizophrenia (ECT+ antipsychotics, $\mathrm{n}=13$; antipsychotics only, $\mathrm{n}=16)$ and controls $(\mathrm{n}=34)$ and tested the ability of these classifier scores to predict reduced PANSS scores after treatment with either ECT or antipsychotics alone. ${ }^{17}$ The classifier score with six functional networks achieved a correct classification rate of $83.82 \%$ [specificity $=91.18 \%$, sensitivity $=76.47 \%$, area under the receiver operating characteristic curve $(\mathrm{AUC})=0.90$ ]. The six networks were the DMN, the MTL, the language network, the corticostriatal network, the frontal-parietal network, and the cerebellum. Changes in these classifier scores correlated with improvements in the PANSS scores for the total $(\mathrm{r}=0.71)$, negative $(\mathrm{r}=0.76)$, and general $(\mathrm{r}=0.66)$ scales. Additionally, baseline classifier scores had predictive value, with significant negative associations with the PANSS total $(\mathrm{r}=-0.75)$, positive $(\mathrm{r}=-0.70)$, negative $(\mathrm{r}=-0.78)$, and general subscores $(\mathrm{r}=-0.65)$. Low baseline classifier scores indicated a lower discriminatory power in differentiating between patients with schizophrenia and controls, and were shown to have predictive value for greater ECT responses. Notably, patients with schizophrenia undergoing antipsychotic treatment alone showed a similar pattern, with lower baseline classifier scores predicting larger treatment responses. Additionally, in the ECT group, FC in the posterior cingulate cortex (PCC), left STG, right angular gyrus, and right MTG increased, while FC in the right ACC, left MTG, and right precuneus decreased after ECT.

\section{REFERENCES}

1. Uesugi H, Toyoda J, Iio M. Positron emission tomography and plasma biochemistry findings in schizophrenic patients before and after electroconvulsive therapy. Psychiatry Clin Neurosci 1995;49:131-135.

2. Escobar R, Rios A, Montoya ID, Lopera F, Ramos D, Carvajal C, et al. Clinical and cerebral blood flow changes in catatonic patients treated with ECT. J Psychosom Res 2000;49:423-429.

3. Fujita Y, Takebayashi M, Hisaoka K, Tsuchioka M, Morinobu S, Yamawaki S. Asymmetric alternation of the hemodynamic response at the prefrontal cortex in patients with schizophrenia during electroconvulsive therapy: a near-infrared spectroscopy study. Brain Res 2011;1410:132-140.

4. Gan JL, Duan HF, Cheng ZX, Yang JM, Zhu XQ, Gao CY, et al. Neuroprotective effect of modified electroconvulsive therapy for schizophrenia. J Nerv Ment Dis 2017;205:480-486.

5. Lotfi M, Jahromi MG, Firoozabadi A, Jahromi LR. Effect of adjuvant electroconvulsive therapy compared to antipsychotic medication alone on the brain metabolites of patients with chronic schizophrenia: a proton magnetic resonance spectroscopy study. Iran J Psychiatry 2018;13:215-221.

6. Xia M, Wang J, Sheng J, Tang Y, Li C, Lim K, et al. Effect of electroconvulsive therapy on medial prefrontal $\gamma$-aminobutyric acid among schizophrenia patients. J ECT 2018;34:227-232.

7. Thomann PA, Wolf RC, Nolte HM, Hirjak D, Hofer S, Seidl U, et al. Neuromodulation in response to electroconvulsive therapy in schizophrenia and major depression. Brain Stimul 2017;10:637-644.

8. Wolf RC, Nolte HM, Hirjak D, Hofer S, Seidl U, Depping MS, et al. Structural network changes in patients with major depression and schizophrenia treated with electroconvulsive therapy. Eur Neuropsychopharmacol 2016;26:1465-1474.

9. Jiang Y, Xia M, Li X, Tang Y, Li C, Huang H, et al. Insular changes induced by electroconvulsive therapy response to symptom improvements in schizophrenia. Prog Neuropsychopharmacol Biol Psychiatry 2019;89:254-262.

10. Jiang Y, Xu L, Li X, Tang Y, Wang P, Li C, et al. Common increased hippocampal volume but specific changes in functional connectivity in schizophrenia patients in remission and non-remission following electroconvulsive therapy: a preliminary study. Neuroimage Clin 2019;24:102081.

11. Wang J, Tang Y, Curtin A, Xia M, Tang X, Zhao Y, et al. ECT-induced brain plasticity correlates with positive symptom improvement in schizophrenia by voxel-based morphometry analysis of grey matter. Brain Stimul 2019;12:319-328.

12. Gong J, Cui LB, Xi YB, Zhao YS, Yang XJ, Xu ZL, et al. Predicting response to electroconvulsive therapy combined with antipsychotics in schizophrenia using multi-parametric magnetic resonance imaging. Schizophr Res 2019;216:262-271.

13. Sambataro F, Thomann PA, Nolte HM, Hasenkamp JH, Hirjak D, Kubera KM, et al. Transdiagnostic modulation of brain networks by electroconvulsive therapy in schizophrenia and major depression. Eur Neuropsychopharmacol 2019;29:925-935.

14. Huang H, Jiang Y, Xia M, Tang Y, Zhang T, Cui H, et al. Increased resting-state global functional connectivity density of default mode network in schizophrenia subjects treated with electroconvulsive therapy. Schizophr Res 2018;197:192-199.

15. Wang J, Jiang Y, Tang Y, Xia M, Curtin A, Li J, et al. Altered functional connectivity of the thalamus induced by modified electroconvulsive therapy for schizophrenia. Schizophr Res 2020;218:209-218.

16. Yang X, Xu Z, Xi Y, Sun J, Liu P, Liu P, et al. Predicting responses to electroconvulsive therapy in schizophrenia patients undergoing antipsychotic treatment: Baseline functional connectivity among regions with strong electric field distributions. Psychiatry Res 2020;299:111059.

17. Li P, Jing RX, Zhao RJ, Ding ZB, Shi L, Sun HQ, et al. Electroconvulsive therapy-induced brain functional connectivity predicts therapeutic efficacy in patients with schizophrenia: a multivariate pattern recognition study. NPJ Schizophr 2017;3:21. 
Supplementary Table 1. Characteristics of the studies included in the review

\begin{tabular}{|c|c|}
\hline Author, year/modality & Study participants \\
\hline $\begin{array}{l}\text { Uesugi et al., 19951 } \\
\text { PET }\end{array}$ & $\begin{array}{l}5 \mathrm{SZ} \text { (mean age: 41.4, mean DOI: 22.6) } \\
6 \mathrm{HC}\end{array}$ \\
\hline $\begin{array}{l}\text { Escobar et al., } 2000^{2} \\
\text { SPECT }\end{array}$ & $\begin{array}{l}5 \mathrm{SZ} \text { with catatonia (mean age: } 29.4 \text { ) } \\
4 \mathrm{MDD} \text { with catatonia (mean age: } 27 \text { ) }\end{array}$ \\
\hline $\begin{array}{l}\text { Fujita et al., } 2011^{3} \\
\text { NIRS }\end{array}$ & $\begin{array}{l}11 \mathrm{SZ} \text { (mean age: 45.8, DOI: 31.1) } \\
10 \text { depression (mean age: 64.5, DOI: 7.5) }\end{array}$ \\
\hline $\begin{array}{l}\text { Gan et al., } 2017^{4} \\
\text { MRS }\end{array}$ & $\begin{array}{l}\text { Randomized } \\
\text { 32 SZ (ECT) (mean age: 27.5, } \\
\text { DOI: 9.8, age at onset: 26.7) } \\
\text { 34 SZ (antipsychotics; AP) } \\
\text { (mean age: 27.8, DOI: 10.0, age at onset: 26.9) } \\
34 \mathrm{HC} \text { (mean age: 26.7) }\end{array}$ \\
\hline
\end{tabular}

\begin{tabular}{|c|c|}
\hline Measurement & ECT protocol \\
\hline $\begin{array}{l}\text { Z: rCBF at 1) baseline, 2) 5-17 } \\
\text { 3) 5-6 months after ECT } \\
\text { IC: measured once }\end{array}$ & $\begin{array}{l}\text { Bifrontal ECT (four times per week, } \\
\text { from } 6 \text { to } 24 \text { sessions per patient, } \\
\text { with a mean of } 16.4 \text { sessions) }\end{array}$ \\
\hline
\end{tabular}

ROI: both frontal, temporal, and occipital lobes, cerebellum, caudate, parahippocampus, putamen, and thalamus Z, MDD: one week before ECT and one week after ECT ROI: both frontal, parietal, temporal, and occipital lobes
basal ganglia, thalamus, and cingulum

CBF measured by normalized tissue hemoglobin index (nTHI) and depression: before, during, and affer each ECT session ROI: bilateral prefrontal cortical regions

SZ, MDD: NAA/Cr and Cho/Cr ratio before and after ECT

HC: measured once

ROI: bilateral prefrontal cortices and bilateral thalami

\section{Bitemporal ECT $(3$ times per week}

from 5 to 15 sessions per patient)

Bifrontotemporal ECT

(mean of 6.8 sessions per patient)

MRS was measured after treatment in both groups

(no pretreatment MRS)

ROI: bilateral prefrontal cortices, occipital cortex, thalamus,

hippocampus

SZ: medial prefrontal GABA/Cr concentrations before and

after 4 weeks of treatment

HC: measured once
ROI: medial prefrontal cortex

$17 \mathrm{SZ}$ (AP) (mean age: 31.0, DOI: 7.75$)$

$19 \mathrm{HC}$ (mean age: 30.9 )

GMV, rsFC

SZ, MDD: before and after ECT

HC: measured once

ROI (structural): amygdala, hippocampus, and insula

ctional): right amygdala, right cortical regions.

and hypothalamus

SZ (ECT, AP): prior to ECT and after 6 weeks of treatment Bilateral ECT (gradually tapered from with either $\mathrm{ECT}+\mathrm{AP}$ or AP

HC: at baseline only

gFCD, rsFC

SZ: before and after 4 weeks of treatment

HC: measured once

Whole brain analys

GMV, rsFC

HC: measured once

ROI: insula

SZ, MDD: before and after ECT

HC: measured once
Whole brain analysis

$8 \mathrm{SZ}(\mathrm{ECT})$

$8 \mathrm{MDD}(\mathrm{ECT})$

$20 \mathrm{HC}$

Source-based morphometry

$\mathrm{SZ}, \mathrm{MDD}$ : before and

HC: measured once
Whole brain analysis

$12 \mathrm{MDD}$
$21 \mathrm{HC}$

$\begin{array}{ll}\text { Wang et al., 2019"1 } & 21 \mathrm{SZ} \text { (ECT) } \\ \text { sMRI } & 21 \mathrm{SZ}(\mathrm{AP})\end{array}$

SZ: MRI before 24 hours prior to treatment,

HC: measured once

ROI analysis followed the results from whole brain analysis

SZ: MRI before 24 hours prior to treatment,

ROI: whole hippocampus and hippocampal subfields

SZ: MRI before 24 hours prior to treatment,

MRI 24 to 48 hours after treatment completion

ROI: 16 thalamic subfields as seeds to whole brain

SZ: a day before the start of ECT, a day after the completion

of ECT sessions

ROI: 10 ROIs using baseline $\mathrm{FC}$ modeling

to predict ECT response

SZ: at baseline only (for prediction of ECT effect

via improvement in PANSS)
Bifrontotemporal ECT (3 times per week

SZ: sMRI and fMRI before and after 4 weeks of treatment

MRI 24 to 48 hours after treatment completion
ECT method not specified (at least twice a week for at least 8 sessions)

Bitemporal ECT $(3$ times per week. Number of sessions were determin by individual clinical response Total number of sessions ranged from 8 to 12 with a mean of 11.3)

Right unilateral ECT (3 times per week. Number of sessions were individualized of 11.3 sessions for SZ and 10.8 sessions for MDD)

5/week to 3/week and 1/week)

Bitemporal ECT (3 times per week Number of sessions were individualized until symptom improvement from 8 to 12 sessions, with a mean of 11.5 sessions)

Bitemporal ECT (3 times per week.

Number of sessions were individualized

until symptom improvement from 8 to 12 sessions,

with a mean of 11.5 sessions)

Right unilateral ECT (3 times per week.
Number of sessions were individualized

until symptom improvemen

Mean number of ECT sessions were

Right unilateral ECT (3 times per week)

Bitemporal ECT

(3 times per week for total of 4 weeks)

Bitemporal ECT

( 3 times per week for total of 4 weeks)

Bitemporal ECT
(3 times per week for total of 4 weeks)

Bitemporal ECT ( 3 times per week,

with a mean of 9.15 sessions)

Bitemporal ECT (3 times per week

with a mean of 10.5 sessions)

\section{Study findings}

rCBF was higher in both temporal lobes and the left cerebellum at baseline compared to controls. rCBF in both frontal lobes, the right temporal lobe and the right putamen was lower 5 to 17 days after ECT than baseline. Six months after ECT, howe there were no significant differences in $\mathrm{rCBF}$ in each region compared to baseline in schizophrenia patients. The author suggest higher cerebral blood flow in SZ patients. Additionally, after ECT, 3-methoxy-4-hydroxyphenylglycol (MHPG) dereased, wheras high leve of

Pre-ECT SPECT did not differ between SZ and MDD patients. MDD patients required fewer sessions of ECT, and their clinical mprovement was greater than those with $S Z$ (using Modffied Rogers Scale for measuring severity of catatonia). After a week Pre- and post-SPECT measures were not significantly altered after ECT sessions in SZ group.
Pall, and occipital regions in MDD.

In SZ compared to mood disorder patients, hemodynamic responses following ECT showed asymmetric alterations. After the ictal period, when blood flow increases were measured to be maximal, SZ patients showed significantly increased ratio of region blood flow in the left PFC region compared to the right PFC, which was not same in mood disorder patient

All patients responded to ECT treatment.

Both ECT and AP groups responded to treatment (PANSS). At baseline, NAA/Cr was lower in the patient group compared to the control group. The reduced NAA/Cr normalized after treatment with either ECT or AP. However, NAA/Cr increased after the treatment only in the ECT group and not in the AP group. Measures of Cho/Cr were not significantly different among the groups or after treatment.

NAA/Cr changes in the left PFC positively correlated with age at onset $(r=0.44)$, percentage of PANSS reduction $(r=0.41)$, baseline PANSS total score $(\mathrm{r}=0.37$ ), and stimulus intensity of ECT $(\mathrm{r}=0.35)$ and negatively correlated with the duration of illness $(\mathrm{r}=-0.41)$. NAA/Cr changes in the left thalamus positively correlated with age at onset $(\mathrm{r}=0.33)$ and negatively correlated with duration of illness $(r=-0.35)$. These results suggest that if age at onset is older and the duration of illness shorter, the greater the NAA/Cr will increase after treatment with ECT.

Those who received ECT had increased NAA/Cr in the left PFC compared to those who received only AP treatment. $\mathrm{Cho/Cr}$ in the left PFC and left thalamus were lower in the ECT group than in the AP group. Clinical improvement did not differ between the two groups.

Medial prefrontal GABA levels did not significantly differ when comparing the three groups. However, when two patient groups (ECT and AP) were combined and compared with controls, their prefrontal GABA levels were lower. After treatment, prefronta GABA levels in the ECT and AP groups did not differ. rmANOVA showed a nonsignificant group effect and time by group effect, but a significant time effect of baseline versus treatment. Post hoc paired t-test found significant increases in prefrontal GABA only in the ECT group and not in the AP group.

in not correlate with prefrontal GABA level changes.

In both SZ and MDD groups, GMV in the MTL, comprising the amygdala, anterior part of the hippocampus and insula increase after ECT, but did not correlate with symptom improvement. The GMV increase in the amygdala was inversely correlated with

In SZ patients at baseline, there was significantly reduced GMV in the right insula and right amygdala. After ECT, amygdala volume did not differ with the control group, and insula GMV was greater in the SZ group than the control group. GMV
in the MDD group did not differ from the control group at baseline, but after ECT, the MDD group showed increased amygdala volumes that exceeded the control group.

ECT reduced rsFC between the right amygdala, right temporoparietal junction, right medial PFC, right and left posterior insula and right DLPFC. rsFC between the right amygdala and hypothalamus increased after ECT, and this change was inversely correlated with baseline connectivity strength of the same region. Post hoc comparison showed rsFC increase between the right amygdala and hypothalamus was significant in SZ after ECT. However, these changes did not correlate with clinical improvements Authors built a classification model using rsFC to differentiate between schizophrenia subgroups and controls. The six networks included were the DMN, MTL, language network, corticostriatal network, frontal-parietal network, and cerebellum. In the ECT group, FC in the PCC, left STG, right angular gyrus, and right MTG were increased, while FC in the right ACC, left MTG, and right precuneus were decreased after ECI.

Repeated ANCOVA revealed significant differential effects of group by time in the left precuneus, $\mathrm{vmPFC}$, and dmPFC. Post hoc analysis showed that $\mathrm{gFCD}$ was increased after $\mathrm{ECT}$ compared to baseline in the $\mathrm{dmPFC}$, vmPFC, and left precuneus. Howeve these changes did not correlate with clinical improvements. In the AP-only group, no significant changes were observed after treatment compared to baseline.

In the ECT group, GMV in the bilateral posterior insula was increased after treatment, and this change correlated with symptom

improvements. In the AP group, GMV in the insular subregions were reduced after treatment.
After ECT, decreased functional connectivity between the right posterior insula and left orbitofrontal cortex and the left posterior insula and middle occipital gyrus was observed, which also correlated with symptom improvements.

Patients had reduced connectivity within the striatothalamic network (in the thalamus) and increased low-frequency oscillation in the striatal network. ECT reduced low-frequency oscillations in the striatal network and increased functional connectivity in the medial PFC within the DMN. Additionally, after ECT, FC of the executive network and the DMN was reduced and FC of the executive network and the salience network was increased.

MDD: Prior to ECT, GMV in the ACC/MPFC, bilateral thalami, and MTL network was smaller. After ECT, structural network strength of the ACC/MPFC and MTL increased but did not correlate with symptom improvements (HAM-D)

SZ: Hio to $\mathrm{ECT}, \mathrm{GMV}$ he cholo

Comparing among the three groups, a significant group by time effect was found in four brain regions: left parahippocampal gyrus/ hippocampus, right parahippocampal gyrus/hippocampus, right temporal pole and mid/superior temporal gyrus, and right ECT and AP groups showed no significant differences in terms of GMV when compred to controls at both baseline and posttreatment.

ECT group showed a significant positive correlation of GMV changes in the right parahippocampal gyrus/hippocampus with reductions in positive subscores on the PANSS.

Compared to the AP group, the ECT group had increased bilateral hippocampal volume. Both responders and nonresponders
showed increased hippocampal volume In nonresponders lower baseline volume in the hipposamps was found. Increased FC between the hippocampus and the PFC and the default mode network were found in ECT responders.

Significant group by time effect in $\mathrm{FC}$ of the right thalamus to right putamen. Post hoc analyses showed increased $\mathrm{FC}$ between right thalamus to right putamen in the ECT group compared to the AP group. After 4 weeks, FC of thalamus to sensory cortex increased in the ECT group, while FC decreased in the AP group. Using hypothetical simulation of strong electric fields during ECT stimulus and models to predict ECT response utilizing baseline the 10 ROIs, FC between the right amygdala and left hippocampus was significantly decreased.

Aiming to use multiparametric markers of MRI to predict effects of ECT, the authors integrated baseline gray matter (GM) features and white matter features (WM). Selection of GM and WM tract ROIs were performed by simulation of electrical fields during ECT stimulus by modeling, and those regions with electrical field strength larger than $35 \mathrm{~V} / \mathrm{m}$ were included (23 GM ROIs, 37 WM tracts). Models were tested to predict difference in PANSS scores prior and after ECT. GM features included the left IFG, right insula, left MTG, and right STG, and the WM tracts between the left calcarine-left superior temporal pole, right lingual-right ITG, left MOG- left ITG, right MTG-right ITG, and right IFG-right insula.

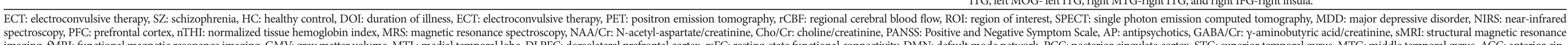

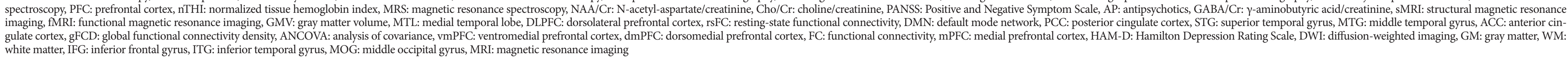


Findings

NAA/Cr changes in the left PFC (positive: age at onset, percentage of PANSS reduction, baseline PANSS total score, stimulus intensity of ECT; negative: duration of illness) ${ }^{4}$

NAA/Cr changes in the left thalamus (positive: age at onset; negative: duration of illness) ${ }^{4}$

GMV increase in the left DLPFC (positive: improvement in PANSS total score) ${ }^{8}$

Baseline GMV in the amygdala (negative: post-ECT GMV increase in amygdala) ${ }^{7}$

Volume increase in the right parahippocampal gyrus/hippocampus (positive: reduction in positive subscores on the PANSS) ${ }^{11}$

GMV increase in the right posterior insula (positive: PANSS total, positive, and general subscore reductions) ${ }^{9}$

Increase in right amygdala-hypothalamus connectivity (negative: baseline connectivity strength of the same region)

FC between the left posterior insula and the left MOG (positive: PANSS general and negative subscore reductions) ${ }^{9}$

FC between the right posterior insula and left OFC (positive: PANSS negative subscore reductions) ${ }^{9}$

Lower classifier scores constituted from six functional networks at baseline predicted greater ECT and antipsychotics response (the DMN, the MTL, the language network,

the corticostriatal network, the frontal-parietal network, and the cerebellum) (positive: changes in PANSS total, negative, and general subscores) ${ }^{17}$

$\uparrow$ lt. HATA volumes in responders compared with nonresponders ${ }^{10}$

Increase in volume in CA4 subfield of the hippocampus (positive: PANSS general subscore reductions) ${ }^{10}$

FC between the left caudal hippocampus and right angular gyrus (positive: PANSS general subscore reductions) ${ }^{10}$

No significant association of FC change in the thalamic subfields and PANSS score changes ${ }^{15}$

rsFC between the rt. amygdala and lt. hippocampus as predictive features for ECT response ${ }^{16}$

Decrease in FC between amygdala and hippocampus (positive: percentage reduction in PANSS total scores) ${ }^{16}$

Selected 10 features (GMV, WM) of the final predictive model which included the baseline measures of the right insula and the left IFG (associations with treatment response measured by changes in PANSS total scores) $)^{12}$

ECT: electroconvulsive therapy, NAA/Cr: N-acetylaspartate/creatinine, PFC: prefrontal cortex, PANSS: Positive and Negative Symptom scale, GMV: gray matter volume, DLPFC: dorsolateral prefrontal cortex, FC: functional connectivity, MOG: middle occipital gyrus, OFC: orbitofrontal cortex, DMN: default mode network, MTL: medial temporal lobe, HATA: hippocampusamygdala transition area, CA4: cornu ammonis 4, rsFC: resting-state functional connectivity, rt.: right, lt.: left, WM: white matter, IFG: inferior frontal gyrus 


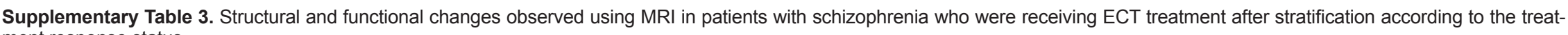
ment response status

\begin{tabular}{|c|c|c|}
\hline & Responders & Nonresponders \\
\hline Baseline & $\begin{array}{l}\text { Lower classifier scores comprised of six functional networks at baseline predictec } \\
\text { the corticostriatal network, the frontal-parietal network, and the cerebellum })^{17} \\
\uparrow \text { lt. HATA volumes in responders compared with nonresponders }{ }^{10} \\
\text { rsFC between the rt. amygdala and lt. hippocampus was a predictor of the ECT r } \\
\text { GMV features at baseline in the lt. IFG, rt. insula, lt. MTG, and rt. STG predicted } \\
\text { WM tract features at baseline in the lt. calcarine-lt. sup. temporal pole, rt. lingual } \\
\text { to ECT }{ }^{12}\end{array}$ & $\begin{array}{l}\text { greater ECT and antipsychotic responses (the DMN, the MTL, the language network, } \\
\text { sponse }^{16} \\
\text { he response to ECT }{ }^{12} \\
\text { rt. ITG, lt. MOG- lt. ITG, rt. MTG-rt. ITG, and rt. IFG-rt. insula predicted the response }\end{array}$ \\
\hline After ECT & $\begin{array}{l}\uparrow \mathrm{rsFC} \text { between } 1) \text { the hippocampus and PFC and between } 2 \text { ) the hippocampus } \\
\text { and DMN in responders after ECT } \\
\uparrow \text { rsFC between } 1 \text { ) the PPtha.R and rt. inf. temporal cortex and 2) PPtha.R and } \\
\text { rt. cerebellum in responders after ECT }{ }^{15}\end{array}$ & $\begin{array}{l}\downarrow \text { rsFC between the hippocampus and primary sensory network in nonresponders } \\
\text { after ECT } \\
\downarrow \text { rsFC between 1) the PPtha.R and rt. inf. temporal cortex, 2) PPtha.R and rt. cerebellum, } \\
\text { 3) PPtha.R and rt. precuneus, and 4) PPtha.R and lt. cerebellum in nonresponders } \\
\text { after ECT }\end{array}$ \\
\hline
\end{tabular}

MRI: magnetic resonance imaging, ECT: electroconvulsive therapy, DMN: default mode network, MTL: medial temporal lobe, lt.: left, HATA: hippocampus-amygdala transition area, rsFC:

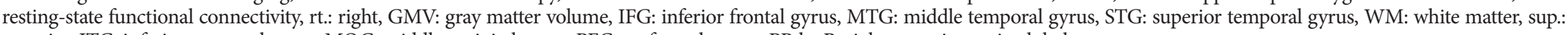
superior, ITG: inferior temporal gyrus, MOG: middle occipital gyrus, PFC: prefrontal cortex, PPtha.R: right posterior parietal thalamus 